\title{
$E 1$ and $M 1$ radiative transitions involving heavy-light axial, pseudoscalar, and vector quarkonia in the framework of the Bethe-Salpeter equation
}

\author{
Vaishali Guleria $\odot,{ }^{1}$ Eshete Gebrehana $\odot,{ }^{2}$ and Shashank Bhatnagar $\odot^{1, *}$ \\ ${ }^{1}$ Department of Physics, University Institute of Sciences, Chandigarh University, Mohali-140413, India \\ ${ }^{2}$ Department of Physics, Woldia University, P.O.Box 400, Woldia, Ethiopia
}

(Received 6 July 2021; accepted 14 October 2021; published 29 November 2021; corrected 2 December 2021)

\begin{abstract}
In the present work, we calculate $M 1$ transitions, $0^{-+} \rightarrow 1^{--} \gamma$, and $E 1$ transitions involving axial vector mesons such as $1^{+-} \rightarrow 0^{-+} \gamma$ and $0^{-+} \rightarrow 1^{+-} \gamma$ for which very little data is available as of now. We make use of the general structure of the transition amplitude $M_{\mathrm{fi}}$ expressed as a linear superposition of terms involving all possible combinations of ++ and -- components of Salpeter wave functions of final and initial hadrons. In the present work, we make use of two leading Dirac structures in the hadronic BetheSalpeter wave functions of the involved hadrons, which makes the formulation more rigorous. We evaluate the decay widths for both the above mentioned $M 1$ and $E 1$ transitions. We have used algebraic forms of Salpeter wave functions obtained through analytic solutions of mass spectral equations for ground and excited states of $1^{--}, 0^{-+}$, and $1^{+-}$heavy-light quarkonia in an approximate harmonic oscillator basis to do analytic calculations of their decay widths. We have compared our results with experimental data, where ever available, and other models.
\end{abstract}

DOI: 10.1103/PhysRevD.104.094045

\section{INTRODUCTION}

One of the challenging areas in hadronic physics is probing the inner structure of hadrons. Charmonium occupies an intermediate regime between the $b \bar{b}$ system and the light mesons. Radiative decays of charmonia are good testing grounds for various models, due to the fact that an emitted photon can be directly detected, and electromagnetic interactions are well understood.

Radiative transitions characterized by $\Delta L=0$ are the magnetic dipole, $M 1$ transitions, while those characterized by $|\Delta L|=1$ are the electric dipole, $E 1$ transitions. The $M 1$ transition mode is sensitive to relativistic effects, esspecially between different spatial multiplets (where $n>n^{\prime}$ ), while the $E 1$ transitions are much stronger than $M 1$ transitions, and involve transitions between excited states. In this work, besides the $M 1$ transitions, we study the $E 1$ transitions involving $1^{+-}$mesons, which are the $P$-wave states.

The $P$-wave $c \bar{c}$ states were first observed in 1976 by the SLAC-LBL experiment at SLAC/SPEAR [1,2], where they observed the decay $\Psi(3684) \rightarrow \gamma+\chi_{c}$. The $P$-wave $b \bar{b}$

\footnotetext{
* Corresponding author. shashank_bhatnagar@yahoo.com

Published by the American Physical Society under the terms of the Creative Commons Attribution 4.0 International license. Further distribution of this work must maintain attribution to the author(s) and the published article's title, journal citation, and DOI. Funded by SCOAP ${ }^{3}$.
}

states were first observed by the Columbia-Stony Brook (CUSB) experiment at the Cornell CESR electron-positron storage ring $[3,4]$ and confirmed by the CLEO experiment at CESR [5].

An indirect way of producing $P$-wave states is through $e^{-} e^{+}$annihilation, which produces ${ }^{3} S_{1}\left(1^{--}\right)$charmonium states such as $J / \Psi(1 S)$ and $\Psi(2 S)$. Then the $M 1$ and $E 1$ decays of these states produce charmonium states, ${ }^{1} S_{0}$ $\left(0^{-+}\right)$, such as $\eta_{c}$ and ${ }^{3} P_{1}\left(1^{++}\right)$such as $\chi_{c 1}$, respectively. Now, amongst the charmonia below $D \bar{D}$ threshold, the axial, $h_{c}\left({ }^{1} P_{0}\right)$ is the least accessible. We wish to mention that the $1^{+-}$meson state was first detected in $p \bar{p}$ collisions by the $R 704$ Collaboration [6]. In 1992, E760 reported the observation of the $h_{c}$ in the $J / \Psi \pi^{0}$ decay mode, in the reaction $p \bar{p} \rightarrow h_{c} \rightarrow \pi^{0}+J / \Psi$ at $M_{h_{c}}=3526.2 \pm 0.15 \pm$ $0.2 \mathrm{MeV}$ with $\Gamma_{h_{c}} \leq 1.1 \mathrm{MeV}$ [7].

In 2005, FNAL E760 [8] analyzed two decay modes of $h_{c}$, the $\pi^{0} J / \Psi$ decay mode and the $\eta_{c} \gamma$ decay mode, through the reactions (a) $p \bar{p} \rightarrow h_{c} \rightarrow \pi^{0}+J / \Psi, J / \Psi \rightarrow e^{-} e^{+}, \pi^{0} \rightarrow \gamma \gamma$, and (b) $p \bar{p} \rightarrow h_{c} \rightarrow \eta_{c} \gamma, \eta_{c} \rightarrow \gamma \gamma$, using data for both runs. They found statistically significant enhancement with mass $M_{h_{c}}=3525.8 \pm 0.2 \pm 0.2 \mathrm{MeV}$ and $\Gamma_{h_{c}}<1 \mathrm{MeV}$. The observation of $h_{c}$ is important since it provides information on the spin dependence of quark-antiquark interactions. However, the best clue for $h_{c}(1 P)$ came from the CLEO Collaboration from isospin violating transition $e+\bar{e} \rightarrow$ $\Upsilon(2 S) \rightarrow h_{c}+\pi^{0}$ [9]. And very recently, the BES-III Collaboration reported $h_{c}$ production in the process $e^{+} e^{-} \rightarrow$ $\pi^{+} \pi^{-} h_{c}$ [10]. 
The $1^{++}$mesons are seen in $p p$ collisions. However, not many decays of these mesons are experimentally observed as can be checked from Particle Data Group (PDG) tables [11,12].

The $M 1$ and $E 1$ transitions of charmonia (that includes axial quarkonia) are quite interesting, and have been recently studied in various models, such as relativistic quark models (RQM) [13,14], effective field theory $[15,16]$, light-front quark models [17-19], lattice QCD [20,21], Bethe-Salpeter equation [22-26], and potential models [27].

In a recent work [26], we calculated the radiative $M 1$ decays $1^{--} \rightarrow 0^{-+} \gamma$ and radiative $E 1$ decays involving scalar mesons $\left({ }^{1} P_{1}\right)$ such as $0^{++} \rightarrow 1^{--} \gamma$ and $1^{--} \rightarrow 0^{++} \gamma$. In the present paper, we focus on the $E 1$ decays involving axial $\left(1^{+-}\right)$quarkonia and the $M 1$ decays of pseudoscalar charmonia $0^{-+} \rightarrow 1^{--} \gamma$. Thus, in this work, we study $E 1$ radiative transitions involving these axial mesons through processes such as $1^{+-} \rightarrow 0^{-+} \gamma\left(\right.$ such as $\left.h_{c} \rightarrow \eta_{c} \gamma\right), 0^{-+} \rightarrow$ $1^{+-} \gamma$ [such as $\eta_{c}(2 S) \rightarrow h_{c} \gamma$ ], along with $M 1$ transitions $0^{-+} \rightarrow 1^{--} \gamma$ [such as $\eta_{c}(2 S) \rightarrow J / \Psi \gamma$ ], which have been studied by some models for which experimental data $[11,12]$ are available for only some of the transitions. The transitions involving leptonic and radiative decays of axial vector quarkonia would also serve as a test for the wave functions of these mesons calculated analytically by solving their mass spectral equations [28] in a recent work.

We wish to mention that decay rates of $M 1$ transitions are much weaker than the rates for $E 1$ transitions. But $M 1$ decay rates are interesting as they allow access to spinsinglet states that are very difficult to produce. Thus, as regards $M 1$ decays, we study decays $\eta_{c}(2 S) \rightarrow J / \Psi(1 S) \gamma$ and $B_{c}(2 S) \rightarrow B_{c}^{*}(1 S) \gamma$. The $B_{c}$ meson was discovered in $p \bar{p}$ collisions at $\sqrt{s}=1.8 \mathrm{TeV}$ using the collider detector at Fermilab tevatron. It is the only heavy meson with two heavy quarks with different flavors that forbid their decays into two photons.

We calculate the radiative decay widths of these heavylight quarkonia for the above mentioned processes in the framework of a $4 \times 4$ Bethe-Salpeter equation (BSE), which is a fully relativistic approach that incorporates the relativistic effect of quark spins and can also describe internal motion of constituent quarks within the hadron in a relativistically consistent manner, due to its covariant structure [29,30]. Our wave functions satisfy the 3D BSE, which is in turn obtained from 3D reduction of the 4D BSE under covariant instantaneous ansatz (CIA) (which is a Lorentz-invariant generalization of instantaneous approximation), and already have relativistic effects. Further, our transition amplitudes also have a relativistically covariant form.

The present work, where we make use of two leading Dirac structures in the structure of BS wave functions of $P\left(0^{-+}\right), V\left(1^{--}\right)$, and $A^{-}\left(1^{+-}\right)$quarkonia involved in these radiative transitions, is more rigorous than our previous work in [26], where we made use of only the most leading Dirac structure $\left(\gamma_{5}\right.$ for $P$ mesons, $i \gamma . \epsilon$ for $V$ meson, and $I$ for $S$ meson) in the BS wave functions of the hadrons involved in the processes.

Now, as mentioned in our previous works [30-34], we are not only interested in studying the mass spectrum of hadrons, which no doubt is an important element to study the dynamics of hadrons, but also the hadronic wave functions that play an important role in the calculation of decay constants, form factors, structure functions, etc. for $Q \bar{Q}$ and $Q \bar{q}$ hadrons. These hadronic Bethe-Salpeter wave functions were calculated algebraically by us in [30-32]. The plots of these wave functions [32] show that they can provide information not only about the long distance nonperturbative physics but also act as a bridge between the long distance and short distance physics, and provide information about the contribution of the short ranged Coulomb interactions in the mass spectral calculation of heavy-light quarkonia. These wave functions can also lead to studies on a number of processes involving $Q \bar{Q}$ and $Q \bar{q}$ states, and provide a guide for future experiments.

This paper is organized as follows: In Sec. II, we give the general formulation of the process $H \rightarrow H^{\prime}+\gamma$ in the framework of a $4 \times 4$ Bethe-Salpeter equation under the covariant instantaneous ansatz. In Sec. III, we calculate the single photon decay widths for the process $P \rightarrow V \gamma$. In Sec. IV, we deal with the process $A^{-} \rightarrow P \gamma$. In Sec. V, we deal with the process $P \rightarrow A^{-} \gamma$, where $P, A^{-}$, and $V$ are the pseudoscalar, axial vector, and vector heavy-light quarkonium states. In Sec. VI, we give numerical results and discussions.

\section{RADIATIVE DECAY PROCESS $\boldsymbol{H} \rightarrow \boldsymbol{H}^{\prime} \gamma$ IN $4 \times 4$ BSE UNDER COVARIANT INSTANTANEOUS ANSATS}

A quark-antiquark bound state system can be described by a Bethe-Salpeter equation

$S_{F}^{-1}\left(p_{1}\right) \Psi(P, q) S_{F}^{-1}\left(-p_{2}\right)=i \int \frac{d^{4} q^{\prime \prime}}{(2 \pi)^{4}} K\left(q, q^{\prime \prime}\right) \Psi\left(P, q^{\prime \prime}\right)$,

where $p_{1}$ and $p_{2}$ are the momenta of the two particles, with the internal momentum of the hadron being $q$ and external hadron momentum $P$ and mass $M$. In Eq. (1), $K\left(q, q^{\prime \prime}\right)$ is the interaction kernel and $S_{F}^{-1}\left( \pm p_{1,2}\right)= \pm i \not \not_{1,2}+m_{1,2}$ are the inverse propagators for the quark and antiquark.

We now make use of the covariant instantaneous ansatz on the BS kernel $K\left(q, q^{\prime \prime}\right)$. Thus we can write $K\left(q, q^{\prime \prime}\right)=K\left(\hat{q}, \hat{q}^{\prime \prime}\right)$, where the BS kernel depends entirely on the component of internal momentum of the hadron $\hat{q}_{\mu}=q_{\mu}-\frac{q . P}{P^{2}} P_{\mu}$, which is a $3 \mathrm{D}$ variable and is orthogonal 
to the total hadron momentum, i.e., $\hat{q} . P=0$, while $\sigma P_{\mu}=\frac{q \cdot P}{P^{2}} P_{\mu}$ is the component of $q$ that is longitudinal to $P$. And the four-dimensional volume element is $d^{4} q=d^{3} \hat{q} M d \sigma$.

It is to be observed that the longitudinal component $M \sigma^{\prime \prime}$ of $q^{\prime \prime}$ does not appear in $K\left(\hat{q}, \hat{q}^{\prime \prime}\right)$. We thus carry out integration over the longitudinal component $M d \sigma^{\prime \prime}$ of the four-dimensional volume element $d^{4} q^{\prime \prime}$ on the right side of Eq. (1). Here, we make use of the fact that

$$
\psi\left(\hat{q}^{\prime \prime}\right)=\frac{i}{2 \pi} \int M d \sigma^{\prime \prime} \Psi\left(P, q^{\prime \prime}\right),
$$

leading to the equation

$S_{F}^{-1}\left(p_{1}\right) \Psi(P, q) S_{F}^{-1}\left(-p_{2}\right)=\int \frac{d^{3} \hat{q}^{\prime \prime}}{(2 \pi)^{3}} K\left(\hat{q}, \hat{q}^{\prime \prime}\right) \psi\left(\hat{q}^{\prime \prime}\right)=\Gamma(\hat{q})$,

where $\Gamma(\hat{q})$ is the hadron-quark vertex function and is directly related to the 4D wave function $\Psi(P, q)$. Multiplying the previous equation from the left by $S_{F}\left(p_{1}\right)$, and from the right by $S_{F}\left(-p_{2}\right)$, we can express the 4D BS wave function $\Psi(P, q)$ in terms of $\Gamma(\hat{q})$ as

$$
\Psi(P, q)=S_{1}\left(p_{1}\right) \Gamma(\hat{q}) S_{2}\left(-p_{2}\right),
$$

where the 4D hadron-quark vertex, that enters into the definition of the 4D BS wave function in the previous equation, is

$$
\Gamma(\hat{q})=\int \frac{d^{3} \hat{q}^{\prime \prime}}{(2 \pi)^{3}} K\left(\hat{q}, \hat{q}^{\prime \prime}\right) \psi\left(\hat{q}^{\prime \prime}\right) .
$$

Following a sequence of steps outlined in [30], we get four Salpeter equations which are effective 3D forms of BSE (Salpeter equations) given below:

$$
\begin{aligned}
\left(M-\omega_{1}-\omega_{2}\right) \psi^{++}(\hat{q}) & =\Lambda_{1}^{+}(\hat{q}) \Gamma(\hat{q}) \Lambda_{2}^{+}(\hat{q}), \\
\left(M+\omega_{1}+\omega_{2}\right) \psi^{--}(\hat{q}) & =-\Lambda_{1}^{-}(\hat{q}) \Gamma(\hat{q}) \Lambda_{2}^{-}(\hat{q}), \\
\psi^{+-}(\hat{q}) & =0, \\
\psi^{-+}(\hat{q}) & =0 .
\end{aligned}
$$

In our framework, the component $\hat{q}_{\mu}$ is always orthogonal to $P_{\mu}$ and satisfies the relation $\hat{q} . P=0$, irrespective of whether $q . P=0$ (i.e., $\sigma=0$ ) or $q . P \neq 0$ (i.e., $\sigma \neq 0$ ). Further, due to the Lorentz-invariant nature of $\hat{q}^{2}$, the applicability of this framework of a covariant instantaneous ansatz is valid all the way from low-energy spectra to highenergy transition amplitudes. For details, see $[26,33]$.
We can now introduce projection operators

$$
\begin{aligned}
\Lambda_{j}^{ \pm}(\hat{q}) & =\frac{1}{2 \omega_{j}}\left[\frac{P}{M} \omega_{j} \pm J(j)\left(i m_{j}+\hat{q}\right)\right], \\
J(j) & =(-1)^{j+1}, \quad j=1,2
\end{aligned}
$$

with the relation

$$
\omega_{j}^{2}=m_{j}^{2}+\hat{q}^{2},
$$

and express the 3D BS wave function $\psi(\hat{q})$ in terms of the ,,+++--+ and -- components as

$$
\psi(\hat{q})=\psi^{++}(\hat{q})+\psi^{+-}(\hat{q})+\psi^{-+}(\hat{q})+\psi^{--}(\hat{q}),
$$

where the various components $\psi^{ \pm \pm}(\hat{q})$ are expressible in terms of the projection operators $\Lambda_{j}^{ \pm}(\hat{q})$ defined above as

$$
\psi^{ \pm \pm}(\hat{q})=\Lambda_{1}^{ \pm}(\hat{q}) \frac{P}{M} \psi(\hat{q}) \frac{P}{M} \Lambda_{2}^{ \pm}(\hat{q}) .
$$

The interaction kernel in BSE is taken as a onegluon-exchange type as regards to the color and spin dependence, and thus has a general structure $K\left(\hat{q}, \hat{q}^{\prime}\right)=$ $\left(\frac{1}{2} \lambda_{1} \cdot \frac{1}{2} \lambda_{2}\right) \gamma_{\mu} \times \gamma_{\mu} V\left(\hat{q}, \hat{q}^{\prime}\right)$, where the scalar part of the kernel is written as a sum of the one-gluon-exchange part and the confinement part as $V\left(\hat{q}, \hat{q}^{\prime}\right)=\frac{4 \pi \alpha_{s}}{\left(\hat{q}-\hat{q}^{\prime}\right)^{2}}+\frac{3}{4} \omega_{q \bar{q}}^{2} \int d^{3} r\left(\kappa r^{2}-\right.$ $\left.\frac{C_{0}}{\omega_{0}^{2}}\right) e^{i\left(\hat{q}-\hat{q}^{\prime}\right) \cdot \vec{r}}$ and $\kappa=\left(1+4 \hat{m}_{1} \hat{m}_{2} A_{0} M^{2} r^{2}\right)^{-\frac{1}{2}}$, where $\hat{m}_{1,2}=$ $\frac{1}{2}\left[1 \pm \frac{\left(m_{1}^{2}-m_{2}^{2}\right)}{M^{2}}\right]$ are the Wightman-Garding definitions [33] of masses of individual quarks, which act like momentum partitioning functions for the two quarks in a hadron.

Here, $\omega_{q \bar{q}}^{2}$ is the flavor dependent spring constant $\omega_{q \bar{q}}^{2}=M \omega_{0}^{2} \alpha_{s}\left(M^{2}\right)$, where the presence of a running coupling constant $\alpha_{s}$ in $\omega_{q \bar{q}}^{2}$ provides an explicit QCD motivation to the BSE kernel. Regarding the parameters of the model, $A_{0}=0.01$ is a parameter that is taken to be small, so that the potential gives a smooth transition from nearly harmonic for light $c \bar{u}$ states to almost linear (for $b \bar{b}$ ) systems [32]. The plots of the confining potential alone versus radial separation $r$ for different mesons is given in [32]. Regarding the parameters of the model, $\omega_{0}=$ $0.22 \mathrm{GeV}$ is the spring constant, $C_{0}=0.69$ is a dimensionless constant, while $\frac{C_{0}}{\omega_{0}^{2}}$ plays the role of ground state energy, $\Lambda=0.250 \mathrm{GeV}$ is the QCD length scale, with input quark masses $m_{u}=0.300 \mathrm{GeV}, m_{s}=0.430 \mathrm{GeV}, m_{c}=$ $1.490 \mathrm{GeV}$, and $m_{b}=4.690 \mathrm{GeV}$. In the present work on radiative decays, we use these same input parameters to calculate the single photon decay widths for the above processes. Our previous studies on mass spectral calculations of heavy-light quarkonia $[31,32]$ were used to fit the input parameters of our model. 

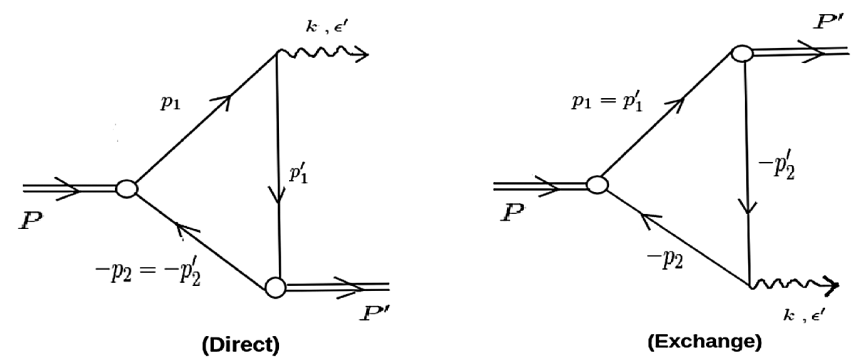

FIG. 1. Radiative decays of heavy-light quarkonia that proceed through direct and exchange diagrams.

In this paper, we study radiative transitions involving single photon decays, such as $H \rightarrow H^{\prime}+\gamma$. This process requires the calculation of a triangle quark-loop diagram. And there are two hadron-quark vertices. The single photon decay of $Q \bar{q}$ quarkonia is described by the direct and exchange Feynman diagrams as shown in Fig. 1.

There are two Lorentz frames involved, the rest frame of the initial meson $H$ and the rest frame of the final meson $H^{\prime}$. Let total momentum and the internal momentum of initial hadron $H$ be designated by $P$ and $q$, while $P^{\prime}$ and $q^{\prime}$ are the corresponding variables of the final hadron $H^{\prime}$. And let $k$ and $\epsilon^{\lambda^{\prime}}$ be the momentum and polarization vectors of the emitted photon, while $\epsilon^{\lambda}$ is the polarization vector of the final meson. Thus if $p_{1,2}$, and $p_{1,2}^{\prime}$ are the momenta of the two quarks in the initial and final hadron respectively, then the kinematical relations between the momenta of initial and final hadrons can be expressed as

$$
\begin{aligned}
P & =p_{1}+p_{2} ; p_{1,2}=\hat{m}_{1,2} P \pm q, \\
P^{\prime} & =p_{1}^{\prime}+p_{2}^{\prime} ; p_{1,2}^{\prime}=\hat{m}_{1,2} P^{\prime} \pm q^{\prime} .
\end{aligned}
$$

From the Feynman diagrams we see that conservation of momentum demands that $P=P^{\prime}+k$. Now, for the first diagram, we have the kinematical relations $p_{1}=p_{1}^{\prime}+k$ and $-p_{2}=-p_{2}^{\prime}$, where $k=P-P^{\prime}$ is the momentum of the emitted photon. And for the second diagram, we have the corresponding relations $p_{1}=p_{1}^{\prime}$ and $-p_{2}^{\prime}=-p_{2}+k$.

Making use of the above equations, the relationship between the internal momenta $q$ and $q^{\prime}$ of the initial and final hadrons in terms of the photon momentum $k$ can be expressed as

$$
\begin{aligned}
& q^{\prime}=q-\hat{m}_{2} k, \\
& q^{\prime}=q+\hat{m}_{1} k,
\end{aligned}
$$

where the first equation is for diagram 1 and second equation is for diagram 2.

For the initial hadron, its internal momentum has already been decomposed as $q=(\hat{q}, i M \sigma)$, where $\hat{q}$ and $M \sigma$ are defined relative to its external momentum $P$. Now, to simplify calculations, since we prefer to work in the rest frame of the initial hadron, we decompose the internal momentum $q^{\prime}$ of the final hadron into two components $q^{\prime}=\left(\hat{q}^{\prime}, i M \sigma^{\prime}\right)$, with $\hat{q}^{\prime}=q^{\prime}-\sigma^{\prime} P$ transverse to the initial hadron momentum $P$ and $\sigma^{\prime}=\frac{q^{\prime} . P}{P^{2}}$ is longitudinal to $P$. Thus, $P . \hat{q}^{\prime}=0$. The relationship between the transverse components of internal momenta of the two hadrons $\hat{q}$ and $\hat{q}^{\prime}$ is [26]

$$
\begin{aligned}
\hat{q}^{\prime} & =\hat{q}+\hat{m}_{2} \hat{P}^{\prime}, \\
\hat{q}^{\prime} & =\hat{q}-\hat{m}_{1} \hat{P}^{\prime}, \\
\hat{P}^{\prime} & =P^{\prime}-\frac{P^{\prime} . P}{P^{2}} P,
\end{aligned}
$$

where the first equation of (13) holds for diagram 1, the second equation holds for diagram 2 , and $\hat{P}^{\prime}$ is the component of the total momentum $P^{\prime}$ of the final hadron transverse to the initial hadron momentum $P$. Here $\hat{q}^{\prime} . P=0$ due to $\hat{P}^{\prime} . P=0$.

In the rest frame of the initial meson, we have $P=(\overrightarrow{0}, i M)$, while for final meson $P^{\prime}=\left(\vec{P}^{\prime}, i E^{\prime}\right)$, where $E^{\prime}=\sqrt{\vec{P}^{\prime 2}+M^{\prime 2}}$, and since the photon momentum can be decomposed as $k=(\vec{k}, i|\vec{k}|)$, where $\vec{k}=-\vec{P}^{\prime}$, since the final meson and photon would be emitted in opposite directions. Hence we get $\left|\vec{P}^{\prime}\right|=|\vec{k}|=\frac{M^{2}-M^{\prime 2}}{2 M}$. Thus the energy of the final meson can be expressed as $E^{\prime}=\frac{M^{2}+M^{\prime 2}}{2 M}$.

The dot products of momenta of the initial and the emitted meson can be expressed as [26]

$$
P^{\prime} . P=-M E^{\prime}=-\frac{M^{2}+M^{\prime 2}}{2} .
$$

Thus, it can be seen that $-E^{\prime}$ acts as the projection of $P^{\prime}$ along the direction of the initial hadron momentum $P$. Similarly, the relationship between the longitudinal components of internal momenta of the two hadrons in the two diagrams can be worked out as [26]

$$
\begin{aligned}
& \sigma^{\prime}=\sigma+\alpha ; \\
& \alpha=\hat{m}_{2} \frac{M^{\prime 2}-M^{2}}{2 M^{2}}(\Rightarrow \text { diagram } 1) \\
& \alpha=-\hat{m}_{1} \frac{M^{\prime 2}-M^{2}}{2 M^{2}}(\Rightarrow \text { diagram } 2),
\end{aligned}
$$

which is again a consequence of the transversality of $\hat{q}^{\prime}$ with initial hadron momentum $P$. Thus, up to Eq. (15), the kinematics are the same for all three processes $(P \rightarrow V \gamma$, $A^{-} \rightarrow P \gamma$, and $\left.P \rightarrow A^{-} \gamma\right)$ studied in this work.

\section{III. $M 1$ RADIATIVE DECAYS $P \rightarrow V \gamma$}

In this section we study the $M 1$ radiative decay process $P \rightarrow V \gamma$, having studied the process $V \rightarrow P \gamma$ in our 
previous work[26]. In the present study, we make use of two leading Dirac structures [as in Eq. (35)] in the BS wave functions of the two hadrons involved in the process, in contrast to a single most leading Dirac structure $\left(\gamma_{5}\right.$ for $P$ meson, and $i \gamma . \epsilon$ for $V$ meson) used in our previous work [26]. This makes the calculations more involved than with use of the single most leading Dirac stricture in the 4D hadronic BS wave functions. It is to be noted that a 4D BS wave function of the initial pseudoscalar meson involved in the process is $\Psi_{P}(P, q)=S_{F}\left(p_{1}\right) \Gamma_{P}(\hat{q}) S_{F}\left(-p_{2}\right)$. Since for transition amplitude calculation we choose to do the calculation in the rest frame of the initial pseudoscalar meson, we write the wave function of the final vector meson as

$$
\Psi_{V}\left(P^{\prime}, q^{\prime}\right)=S_{F}\left(p_{1}^{\prime}\right) \Gamma_{V}\left(\hat{q}^{\prime}\right) S_{F}\left(-p_{2}^{\prime}\right)
$$

where as defined earlier $\hat{q}^{\prime}=q^{\prime}-\frac{q^{\prime} . P}{P^{2}} P$ is transverse to the initial hadron momentum $P$, where the hadron-quark vertex function $\Gamma_{V}\left(\hat{q}^{\prime}\right)$ for the final meson is

$$
\Gamma_{V}\left(\hat{q}^{\prime}\right)=\int \frac{d^{3} \hat{q}^{\prime \prime \prime}}{(2 \pi)^{3}} K\left(\hat{q}^{\prime}, \hat{q}^{\prime \prime \prime}\right) \psi\left(\hat{q}^{\prime \prime \prime}\right) .
$$

Similarly for the final meson, the expression for the 3D BS wave function $\psi\left(\hat{q}^{\prime}\right)$ is expressed in terms of the projection operators as

$$
\psi\left(\hat{q}^{\prime}\right)=\psi^{++}\left(\hat{q}^{\prime}\right)+\psi^{+-}\left(\hat{q}^{\prime}\right)+\psi^{-+}\left(\hat{q}^{\prime}\right)+\psi^{--}\left(\hat{q}^{\prime}\right)
$$

where

$$
\begin{aligned}
\psi^{ \pm \pm}\left(\hat{q}^{\prime}\right) & =\Lambda_{1}^{ \pm}\left(\hat{q}^{\prime}\right) \frac{P}{M} \psi\left(\hat{q}^{\prime}\right) \frac{P}{M} \Lambda_{2}^{ \pm}\left(\hat{q}^{\prime}\right), \\
\Lambda_{j}^{ \pm}\left(\hat{q}^{\prime}\right) & =\frac{1}{2 \omega_{j}^{\prime}}\left[\frac{P}{M} \omega_{j}^{\prime} \pm J(j)\left(i m_{j}+\hat{q}^{\prime}\right)\right],
\end{aligned}
$$

with the relation $\omega_{1,2}^{\prime 2}=m_{1,2}^{2}+\hat{q}^{\prime 2}$.

We can express the electromagnetic transition amplitude of the process as a single integral over $d^{4} q$. Thus this fourdimensional volume integral involves integration over all four components of $q$. To perform this integral, we make use of decomposition of the 4D volume element as $d^{4} q=$ $d^{3} \hat{q} M d \sigma$ using covariant instantaneous ansatz. We will first perform integration over the time part $M d \sigma$, using pole integration methods in the complex $\sigma$ plane, and then express $M_{\mathrm{fi}}$ as an integration over $d^{3} \hat{q}$, which is then numerically worked out. This procedure of integration over $d^{4} q$ is followed in the steps below.

We first write $M_{\mathrm{fi}}$ as

$$
\begin{aligned}
M_{\mathrm{fi}}= & -\int \frac{d^{4} q}{(2 \pi)^{4}} \operatorname{Tr}\left[e_{q} \bar{\Psi}_{V}\left(P^{\prime}, q^{\prime}\right) \mathfrak{\ell}^{\lambda^{\prime}} \Psi_{P}(P, q) S_{F}^{-1}\left(-p_{2}\right)\right. \\
& \left.+e_{\bar{Q}} \bar{\Psi}_{V}\left(P^{\prime}, q^{\prime}\right) S_{F}^{-1}\left(p_{1}\right) \Psi_{P}(P, q) \hat{\ell}^{\lambda^{\prime}}\right] .
\end{aligned}
$$

Here, the first term corresponds to the first diagram, where the photon is emitted from the quark, while the second term corresponds to the second diagram where the photon is emitted from the antiquark, and $e_{q}$ and $e_{Q}$ are the electric charge of the quark and antiquark, respectively, while $\epsilon_{\mu}^{\lambda^{\prime}}$ is the polarization vector of the emitted photon.

We then express Eq. (20) as

$$
\begin{aligned}
M_{\mathrm{fi}}= & -e_{q} \int \frac{d^{3} \hat{q}}{(2 \pi)^{3}} \int \frac{i M d \sigma}{(2 \pi)} \operatorname{Tr}\left[\bar{\Gamma}_{V}\left(\hat{q}^{\prime}\right) S_{F}\left(p_{1}^{\prime}\right) \ell^{\prime} S_{F}\left(p_{1}\right)\right. \\
& \left.\times \Gamma_{P}(\hat{q}) S_{F}\left(-p_{2}\right)\right] \\
& -e_{Q} \int \frac{d^{3} \hat{q}}{(2 \pi)^{3}} \int \frac{i M d \sigma}{(2 \pi)} \operatorname{Tr}\left[\bar{\Gamma}_{V}\left(\hat{q}^{\prime}\right) S_{F}\left(p_{1}\right)\right. \\
& \left.\times \Gamma_{P}(\hat{q}) S_{F}\left(-p_{2}\right) \epsilon^{\prime} S_{F}\left(-p_{2}^{\prime}\right)\right] .
\end{aligned}
$$

Now, we reduce the above equation to the effective 3D form by integrating over the longitudinal component $M d \sigma$ over the poles of the propagators $S_{F}\left(p_{i}\right)$, that are expressed as [26]

$$
\begin{aligned}
S_{F}\left(p_{1}\right) & =\frac{\Lambda_{1}^{+}(\hat{q})}{M \sigma+\hat{m}_{1} M-\omega_{1}+i \epsilon}+\frac{\Lambda_{1}^{-}(\hat{q})}{M \sigma+\hat{m}_{1} M+\omega_{1}-i \epsilon}, \\
S_{F}\left(-p_{2}\right) & =\frac{-\Lambda_{2}^{+}(\hat{q})}{-M \sigma+\hat{m}_{2} M-\omega_{2}+i \epsilon}+\frac{-\Lambda_{2}^{-}(\hat{q})}{-M \sigma+\hat{m}_{2} M+\omega_{2}-i \epsilon}, \\
S_{F}\left(p_{1}^{\prime}\right) & =\frac{\Lambda_{1}^{+}\left(\hat{q}^{\prime}\right)}{M \sigma^{\prime}+\hat{m}_{1}\left(-E^{\prime}\right)-\omega_{1}^{\prime}+i \epsilon}+\frac{\Lambda_{1}^{-}(\hat{q})^{\prime}}{M \sigma^{\prime}+\hat{m}_{1}\left(-E^{\prime}\right)+\omega_{1}^{\prime}-i \epsilon}, \\
S_{F}\left(-p_{2}^{\prime}\right) & =\frac{-\Lambda_{2}^{+}\left(\hat{q}^{\prime}\right)}{-M \sigma^{\prime}+\hat{m}_{2}\left(-E^{\prime}\right)-\omega_{2}^{\prime}+i \epsilon}+\frac{-\Lambda_{2}^{-}(\hat{q})^{\prime}}{M \sigma^{\prime}+\hat{m}_{2}\left(-E^{\prime}\right)+\omega_{2}^{\prime}-i \epsilon} .
\end{aligned}
$$


We now put the propagators into Eq. (21) and multiplying this equation from the left by the relation $\frac{\not P}{M} \frac{\not P}{M}=-1=$ $\frac{\not P}{M}\left(\Lambda_{2}^{+}\left(\hat{q}^{\prime}\right)+\Lambda_{2}^{-}\left(\hat{q}^{\prime}\right)\right)$ [35], the transition amplitude corresponding to the first term in Eq. (21) can be expressed as

$$
\begin{aligned}
& M_{\mathrm{fi}}^{1}=-i e \int \frac{d^{3} \hat{q}}{(2 \pi)^{3}}\left[\Omega_{1}+\Omega_{2}+\Omega_{3}+\Omega_{4}\right] \\
& \Omega_{1}=\int \frac{d \sigma}{(2 \pi)} \frac{i}{M^{3}} \operatorname{Tr}\left[\frac{-P \Lambda_{2}^{+}\left(\hat{q}^{\prime}\right) \bar{\Gamma}_{P}\left(\hat{q}^{\prime}\right) \Lambda_{1}^{+}\left(\hat{q}^{\prime}\right) \boldsymbol{\ell}^{\prime} \Lambda_{1}^{+}(\hat{q}) \Gamma_{V}(\hat{q}) \Lambda_{2}^{+}(\hat{q})}{\left[\sigma-\left(-\alpha+\hat{m}_{1} \frac{E^{\prime}}{M}+\frac{\omega_{1}^{\prime}}{M}\right)\right]\left[\sigma-\left(-\hat{m}_{1}+\frac{\omega_{1}}{M}\right)\right]\left[\sigma-\left(\hat{m}_{2}-\frac{\omega_{2}}{M}\right)\right]}\right] \\
& \Omega_{2}=\int \frac{d \sigma}{(2 \pi)} \frac{i}{M^{3}} \operatorname{Tr}\left[\frac{-P \Lambda_{2}^{+}\left(\hat{q}^{\prime}\right) \bar{\Gamma}_{P}\left(\hat{q}^{\prime}\right) \Lambda_{1}^{+}\left(\hat{q}^{\prime}\right) \epsilon^{\prime} \Lambda_{1}^{-}(\hat{q}) \Gamma_{V}(\hat{q}) \Lambda_{2}^{-}(\hat{q})}{\left[\sigma-\left(-\alpha+\hat{m}_{1} \frac{E^{\prime}}{M}+\frac{\omega_{1}^{\prime}}{M}\right)\right]\left[\sigma-\left(-\hat{m}_{1}-\frac{\omega_{1}}{M}\right)\right]\left[\sigma-\left(\hat{m}_{2}+\frac{\omega_{2}}{M}\right)\right]}\right] \\
& \Omega_{3}=\int \frac{d \sigma}{(2 \pi)} \frac{i}{M^{3}} \operatorname{Tr}\left[\frac{-P \Lambda_{2}^{-}\left(\hat{q}^{\prime}\right) \bar{\Gamma}_{P}\left(\hat{q}^{\prime}\right) \Lambda_{1}^{-}\left(\hat{q}^{\prime}\right) \hat{\ell}^{\prime} \Lambda_{1}^{+}(\hat{q}) \Gamma_{V}(\hat{q}) \Lambda_{2}^{+}(\hat{q})}{\left[\sigma-\left(-\alpha+\hat{m}_{1} \frac{E^{\prime}}{M}-\frac{\omega_{1}^{\prime}}{M}\right)\right]\left[\sigma-\left(-\hat{m}_{1}+\frac{\omega_{1}}{M}\right)\right]\left[\sigma-\left(\hat{m}_{2}-\frac{\omega_{2}}{M}\right)\right]}\right] \\
& \Omega_{4}=\int \frac{d \sigma}{(2 \pi)} \frac{i}{M^{3}} \operatorname{Tr}\left[\frac{-P \Lambda_{2}^{+}\left(\hat{q}^{\prime}\right) \bar{\Gamma}_{P}\left(\hat{q}^{\prime}\right) \Lambda_{1}^{-}\left(\hat{q}^{\prime}\right) \ell^{\prime} \Lambda_{1}^{-}(\hat{q}) \Gamma_{V}(\hat{q}) \Lambda_{2}^{-}(\hat{q})}{\left[\sigma-\left(-\alpha+\hat{m}_{1} \frac{E^{\prime}}{M}-\frac{\omega_{1}^{\prime}}{M}\right)\right]\left[\sigma-\left(-\hat{m}_{1}-\frac{\omega_{1}}{M}\right)\right]\left[\sigma-\left(\hat{m}_{2}+\frac{\omega_{2}}{M}\right)\right]}\right]
\end{aligned}
$$

The contour integrations over $M d \sigma$ are performed over each of the four terms taking into account the pole positions in the complex $\sigma$ plane:

$$
\begin{aligned}
& \sigma_{3}^{ \pm}=-\alpha+\hat{m}_{1} \frac{E^{\prime}}{M} \mp \frac{\omega_{1}^{\prime}}{M} \pm i \epsilon, \\
& \sigma_{1}^{ \pm}=-\hat{m}_{1} \mp \frac{\omega_{1}}{M} \pm i \epsilon, \\
& \sigma_{2}^{ \pm}=\hat{m}_{2} \mp \frac{\omega_{2}}{M} \pm i \epsilon .
\end{aligned}
$$

For the second term in Eq. (21), we can write the amplitude $M_{\mathrm{fi}}^{2}$ as

$$
\begin{aligned}
& M_{\mathrm{fi}}^{2}=-e_{Q} \int \frac{d^{3} \hat{q}}{(2 \pi)^{3}}\left[\Omega_{1}^{\prime}+\Omega_{2}^{\prime}+\Omega_{3}^{\prime}+\Omega_{4}^{\prime}\right] \\
& \Omega_{1}^{\prime}=\int \frac{d \sigma}{(2 \pi)} \frac{i}{M^{3}} \operatorname{Tr}\left[\frac{-\Lambda_{2}^{+}\left(\hat{q}^{\prime}\right) \bar{\Gamma}_{V}\left(\hat{q}^{\prime}\right) \Lambda_{1}^{+}\left(\hat{q}^{\prime}\right) P \Lambda_{1}^{+}(\hat{q}) \Gamma_{P}(\hat{q}) \Lambda_{2}^{+}(\hat{q}) \hat{\epsilon}^{\prime}}{\left[\sigma-\left(-\alpha-\hat{m}_{2} \frac{E^{\prime}}{M}-\frac{\omega_{2}^{\prime}}{M}\right)\right]\left[\sigma-\left(-\hat{m}_{1}+\frac{\omega_{1}}{M}\right)\right]\left[\sigma-\left(\hat{m}_{2}-\frac{\omega_{2}}{M}\right)\right]}\right] \\
& \Omega_{2}^{\prime}=\int \frac{d \sigma}{(2 \pi)} \frac{i}{M^{3}} \operatorname{Tr}\left[\frac{-\Lambda_{2}^{-}\left(\hat{q}^{\prime}\right) \bar{\Gamma}_{V}\left(\hat{q}^{\prime}\right) \Lambda_{1}^{-}\left(\hat{q}^{\prime}\right) P \Lambda_{1}^{+}(\hat{q}) \Gamma_{P}(\hat{q}) \Lambda_{2}^{+}(\hat{q}) \hat{\ell}^{\prime}}{\left[\sigma-\left(-\alpha-\hat{m}_{2} \frac{E^{\prime}}{M}+\frac{\omega_{2}^{\prime}}{M}\right)\right]\left[\sigma-\left(-\hat{m}_{1}+\frac{\omega_{1}}{M}\right)\right]\left[\sigma-\left(\hat{m}_{2}-\frac{\omega_{2}}{M}\right)\right]}\right] \\
& \Omega_{3}^{\prime}=\int \frac{d \sigma}{(2 \pi)} \frac{i}{M^{3}} \operatorname{Tr}\left[\frac{-\Lambda_{2}^{+}\left(\hat{q}^{\prime}\right) \bar{\Gamma}_{V}\left(\hat{q}^{\prime}\right) \Lambda_{1}^{+}\left(\hat{q}^{\prime}\right) P \Lambda_{1}^{+}(\hat{q}) \Gamma_{P}(\hat{q}) \Lambda_{2}^{-}(\hat{q}) \hat{\epsilon}^{\prime}}{\left[\sigma-\left(-\alpha-\hat{m}_{2} \frac{E^{\prime}}{M}-\frac{\omega_{2}^{\prime}}{M}\right)\right]\left[\sigma-\left(-\hat{m}_{1}-\frac{\omega_{1}}{M}\right)\right]\left[\sigma-\left(\hat{m}_{2}+\frac{\omega_{2}}{M}\right)\right]}\right] \\
& \Omega_{4}^{\prime}=\int \frac{d \sigma}{(2 \pi)} \frac{i}{M^{3}} \operatorname{Tr}\left[\frac{-\Lambda_{2}^{-}\left(\hat{q}^{\prime}\right) \bar{\Gamma}_{V}\left(\hat{q}^{\prime}\right) \Lambda_{1}^{-}\left(\hat{q}^{\prime}\right) P \Lambda_{1}^{-}\left(\hat{q}^{\prime}\right) \Gamma_{P}(\hat{q}) \Lambda_{2}^{-}(\hat{q}) \hat{\epsilon}^{\prime}}{\left[\sigma-\left(-\alpha+\hat{m}_{2} \frac{E^{\prime}}{M}+\frac{\omega_{2}^{\prime}}{M}\right)\right]\left[\sigma-\left(-\hat{m}_{1}-\frac{\omega_{1}}{M}\right)\right]\left[\sigma-\left(\hat{m}_{2}+\frac{\left.\omega_{2}\right)}{M}\right)\right]}\right]
\end{aligned}
$$

where the rest of the terms are anticipated to be zero on account of the 3D Salpeter equations. The contour integrations over $M d \sigma$ in Eq. (25) are performed over each of the four terms taking into account the pole positions in the complex $\sigma$ plane:

$$
\begin{aligned}
& \sigma_{3}^{ \pm}=-\alpha-\hat{m}_{2} \frac{E^{\prime}}{M} \mp \frac{\omega_{2}^{\prime}}{M} \pm i \epsilon, \\
& \sigma_{1}^{ \pm}=-\hat{m}_{1} \pm \frac{\omega_{1}}{M} \pm i \epsilon, \\
& \sigma_{2}^{ \pm}=\hat{m}_{2} \mp \frac{\omega_{2}}{M} \pm i \epsilon
\end{aligned}
$$

by closing the contour either above or below the real axis in the complex $\sigma$ plane. 
We now make use of the Salpeter equations in variable $\hat{q}$ in Eq. (6), and the Salpeter equations in variable $\hat{q}^{\prime}$ given below. It is to be noted that the Salpeter equations in $\hat{q}^{\prime}$ involve $-E^{\prime}=\frac{P . P^{\prime}}{M}$, which is the projection of $P^{\prime}$ along the direction of the initial momentum $P$, and are given as [35]

$$
\begin{aligned}
\left(-E^{\prime}-\omega_{1}^{\prime}-\omega_{2}^{\prime}\right) \psi^{++}\left(\hat{q}^{\prime}\right) & =\Lambda_{1}^{+}\left(\hat{q}^{\prime}\right) \Gamma\left(\hat{q}^{\prime}\right) \Lambda_{2}^{+}\left(\hat{q}^{\prime}\right), \\
\left(-E^{\prime}+\omega_{1}^{\prime}+\omega_{2}^{\prime}\right) \psi^{--}\left(\hat{q}^{\prime}\right) & =-\Lambda_{1}^{-}\left(\hat{q}^{\prime}\right) \Gamma\left(\hat{q}^{\prime}\right) \Lambda_{2}^{-}\left(\hat{q}^{\prime}\right), \\
\psi^{+-}\left(\hat{q}^{\prime}\right) & =0, \\
\psi^{-+}\left(\hat{q}^{\prime}\right) & =0 .
\end{aligned}
$$

The results of each of these four integrals $\Omega_{1}, \ldots, \Omega_{4}$ whether we close the contour above or below the real $\sigma$ axis comes out to be the same, thereby validating the correctness of the formalism employed. These results of integrals over $d \sigma$ in $\Omega_{1}, \ldots, \Omega_{4}$, are given as $\alpha_{1}, \ldots, \alpha_{4}$ in Eqs. (29).

This leads to the expression for an effective 3D form of transition amplitude $M_{\mathrm{fi}}^{1}$ under covariant instantaneous ansatz for diagram 1 , as

$$
\begin{aligned}
M_{\mathrm{fi}}^{1}= & -i e \int \frac{d^{3} \hat{q}}{(2 \pi)^{3}} \frac{1}{M^{2}} \operatorname{Tr}\left[\alpha_{1} P \bar{\psi}_{V}^{++}\left(\hat{q}^{\prime}\right) \epsilon^{\prime} \psi_{P}^{++}(\hat{q})\right. \\
& +\alpha_{2} P \bar{\psi}_{V}^{++}\left(\hat{q}^{\prime}\right) \ell^{\prime} \psi_{P}^{--}(\hat{q})+\alpha_{3} P \bar{\psi}_{V}^{--}\left(\hat{q}^{\prime}\right) \epsilon^{\prime} \psi_{P}^{++}(\hat{q}) \\
& \left.+\alpha_{4} P \bar{\psi}_{V}^{--}\left(\hat{q}^{\prime}\right) \epsilon^{\prime} \psi_{P}^{--}(\hat{q})\right]
\end{aligned}
$$

where

$$
\begin{aligned}
& \alpha_{1}=\frac{\left[-E^{\prime}-\omega_{1}^{\prime}-\omega_{2}^{\prime}\right]}{\left[\alpha-\hat{m}_{1} \frac{E^{\prime}}{M}+\hat{m}_{2}-\frac{1}{M}\left(\omega_{1}^{\prime}+\omega_{2}\right)\right]}, \\
& \alpha_{2}=\frac{-\left[-E^{\prime}-\omega_{1}^{\prime}-\omega_{2}^{\prime}\right]}{\left[\alpha-\hat{m}_{1}\left(\frac{E^{\prime}}{M}-1\right)-\frac{1}{M}\left(\omega_{1}+\omega_{1}^{\prime}\right)\right]}, \\
& \alpha_{3}=\frac{\left[-E^{\prime}+\omega_{1}^{\prime}+\omega_{2}^{\prime}\right]}{\left[\alpha-\hat{m}_{1}\left(\frac{E^{\prime}}{M}-1\right)+\frac{1}{M}\left(\omega_{1}+\omega_{1}^{\prime}\right)\right]}, \\
& \alpha_{4}=\frac{-\left[-E^{\prime}+\omega_{1}^{\prime}+\omega_{2}^{\prime}\right]}{\left[\alpha-\hat{m}_{1} \frac{E^{\prime}}{M}+\hat{m}_{2}+\frac{1}{M}\left(\omega_{1}^{\prime}+\omega_{2}\right)\right]},
\end{aligned}
$$

and the projected wave functions $\psi^{ \pm \pm}$are taken from the 3D Salpeter equations [32] derived earlier, which for the initial meson in the internal variable $\hat{q}$ are given in Eq. (6).

[Here, the factors $\left(M \pm \omega_{1} \pm \omega_{2}\right)$ that were also present in the numerators of $\alpha$ 's in Eq. (29), as a result of the first two Salpeter equations in the variable $\hat{q}$ in Eqs. (6), get cancelled from the corresponding factors (in the denominator) resulting from contour integrals over $d \sigma$, while the numerators of $\alpha_{1}, \ldots, \alpha_{4}$ come from the Salpeter equations in the variable $\hat{q}^{\prime}$ in Eq. (27).]

Similarly, the expression for an effective 3D form of transition amplitude $M_{\mathrm{fi}}^{2}$ under a covariant instantaneous ansatz can be expressed as

$$
\begin{aligned}
M_{\mathrm{fi}}^{2}= & -e_{Q} \int \frac{d^{3} \hat{q}}{(2 \pi)^{3}} \frac{1}{M^{2}} \operatorname{Tr}\left[\alpha_{1}^{\prime} \bar{\psi}_{V}^{++}\left(\hat{q}^{\prime}\right) P \psi_{P}^{++}(\hat{q}) \boldsymbol{\epsilon}^{\prime}\right. \\
& +\alpha_{2}^{\prime} \bar{\psi}_{V}^{++}\left(\hat{q}^{\prime}\right) P \psi_{P}^{--}(\hat{q}) \boldsymbol{\epsilon}^{\prime}+\alpha_{3}^{\prime} \bar{\psi}_{V}^{--}\left(\hat{q}^{\prime}\right) P \psi_{P}^{++}(\hat{q}) \boldsymbol{\epsilon}^{\prime} \\
& \left.+\alpha_{4}^{\prime} \bar{\psi}_{V}^{--}\left(\hat{q}^{\prime}\right) P \psi_{P}^{--}(\hat{q}) \boldsymbol{\epsilon}^{\prime}\right] .
\end{aligned}
$$

Here, the results of contour integrals over $d \sigma$ in expressions for $\Omega_{1}^{\prime}, \ldots, \Omega_{4}^{\prime}$ in Eqs. (25) following similar procedure as in $\Omega_{1}, \ldots, \Omega_{4}$ are given as $\alpha_{1}^{\prime}, \ldots, \alpha_{4}^{\prime}$ :

$$
\begin{aligned}
\alpha_{1}^{\prime} & =\frac{\left[-E^{\prime}-\omega_{1}^{\prime}-\omega_{2}^{\prime}\right]}{\left[\alpha-\hat{m}_{1}+\hat{m}_{2} \frac{E^{\prime}}{M}+\frac{1}{M}\left(\omega_{1}+\omega_{2}^{\prime}\right)\right]}, \\
\alpha_{2}^{\prime} & =\frac{-\left[-E^{\prime}-\omega_{1}^{\prime}-\omega_{2}^{\prime}\right]}{\left[\alpha+\hat{m}_{2}\left(1+\frac{E^{\prime}}{M}\right)-\frac{1}{M}\left(\omega_{2}+\omega_{2}^{\prime}\right)\right]}, \\
\alpha_{3}^{\prime} & =\frac{\left[-E^{\prime}+\omega_{1}^{\prime}+\omega_{2}^{\prime}\right]}{\left[\alpha+\hat{m}_{2}\left(1+\frac{E^{\prime}}{M}\right)+\frac{1}{M}\left(\omega_{2}+\omega_{2}^{\prime}\right)\right]}, \\
\alpha_{4}^{\prime} & =\frac{-\left[-E^{\prime}+\omega_{1}^{\prime}+\omega_{2}^{\prime}\right]}{\left[\alpha-\hat{m}_{1}+\hat{m}_{2} \frac{E^{\prime}}{M}-\frac{1}{M}\left(\omega_{1}+\omega_{2}^{\prime}\right)\right]} .
\end{aligned}
$$

Thus, we make use of the generalized method for handling quark-triangle diagrams with two hadron-quark vertices in the framework of a $4 \times 4$ BSE under covariant instantaneous ansatz described in [26], by expressing the transition amplitude $M_{\mathrm{fi}}$ as a linear superposition of terms involving all possible combinations of ++ and -components of Salpeter wave functions of final and initial hadrons through,,++++----++-- , and --++ , with each of the four terms being associated with a coefficient $\alpha_{i}(i=1, \ldots, 4)$, which is the result of pole integration in the complex $\sigma$ plane, which should be a feature of relativistic frameworks.

Now, to calculate the amplitude $M_{\mathrm{fi}}$ for the process, we need the ++ and -- components $\psi_{V}^{ \pm \pm}\left(\hat{q}^{\prime}\right)$ for vector and $\psi_{P}^{ \pm \pm}(\hat{q})$ for pseudoscalar mesons, given as

$$
\begin{aligned}
\psi_{P}^{ \pm \pm}(\hat{q}) & =\Lambda_{1}^{ \pm}(\hat{q}) \frac{P}{M} \psi_{P}(\hat{q}) \frac{P}{M} \Lambda_{2}^{ \pm}(\hat{q}), \\
\psi_{V}^{ \pm \pm}\left(\hat{q}^{\prime}\right) & =\Lambda_{1}^{ \pm}\left(\hat{q}^{\prime}\right) \frac{P}{M} \psi_{V}\left(\hat{q}^{\prime}\right) \frac{P}{M} \Lambda_{2}^{ \pm}\left(\hat{q}^{\prime}\right),
\end{aligned}
$$

to calculate which we need the $3 \mathrm{D}$ wave functions $\psi_{P}(\hat{q})$ and $\psi_{V}\left(\hat{q}^{\prime}\right)$. To derive these, we start with the general 4D decomposition of the BS wave functions [36,37]. Using 3D decomposition under covariant instantaneous ansatz, the 
wave function of vector mesons of dimensionality $M$ can be written as $[26,30,31]$

$$
\begin{aligned}
\psi^{V}(\hat{q})= & i M \chi^{\prime} \chi_{1}(\hat{q})+\epsilon P \chi_{2}(\hat{q})+[\hat{\epsilon} \hat{q}-\hat{q} \cdot \epsilon] \chi_{3}(\hat{q}) \\
& -i[P \hat{\epsilon} \hat{\phi}+\hat{q} . \epsilon P] \frac{1}{M} \chi_{4}(\hat{q})+(\hat{q} \cdot \epsilon) \chi_{5}(\hat{q}) \\
& -i \hat{q} \cdot \epsilon \frac{P}{M} \chi_{6}(\hat{q})-i(\hat{q} . \epsilon) \frac{\hat{\phi}}{M} \chi_{7}(\hat{q})+2(\hat{q} \cdot \epsilon) \frac{P \hat{\phi}}{M^{2}} \chi_{8}(\hat{q}),
\end{aligned}
$$

where $\epsilon^{\lambda}$ is the vector meson polarization vector, while for a pseudoscalar meson, the 3D wave function with dimension $M$ can be written as $[26,30,31]$

$$
\psi^{P}(\hat{q})=N_{P}\left[M \phi_{1}(\hat{q})-i P \phi_{2}(\hat{q})+i \hat{\phi} \phi_{3}(\hat{q})+\frac{P \hat{\phi}}{M} \phi_{4}(\hat{q})\right] \gamma_{5} .
$$

However, during calculation of hadronic observables, such as meson masses and leptonic decay constants, it was noticed that some Dirac covariants [37] in the structure of a hadronic BS wave function contribute much more than others. And in accordance with a naive power counting rule in $[33,38,39]$, for pseudoscalar mesons, one could classify Dirac structures $M \gamma_{5}$ and $P \gamma_{5}$ associated with amplitudes $\phi_{1}$ and $\phi_{2}$, respectively, as leading, while those with $\phi_{3}$ and $\phi_{4}$ as subleading. A similar behavior was observed in the case of vector mesons [33,38], where Dirac structures $M \notin$ and $\epsilon P$ associated with $\chi_{1}$ and $\chi_{2}$, respectively, are leading $[33,38]$, while those associated with $\chi_{3}, \ldots, \chi_{8}$ are subleading. A similar observation about the most leading Dirac structures from all the Dirac structures was made by $[40,41]$. Thus to simplify the algebra, we take 3D wave functions with these two leading Dirac structures (the present work with two leading Dirac structures is more involved than our previous work [26], where we took only the most leading Dirac structure in hadronic BS wave functions of $P, V$, and $S$ mesons [26]), on the basis of their maximum contribution to the calculation of masses and leptonic decay constants of individual mesons in $[33,37-39,41]$,

$$
\begin{aligned}
& \psi_{P}(\hat{q})=N_{P}\left[M \gamma_{5}-i P \gamma_{5}\right] \phi_{P}(\hat{q}), \\
& \psi_{V}(\hat{q})=N_{V}[i M \not \epsilon+\not \ell P] \phi_{V}(\hat{q}),
\end{aligned}
$$

with the 3D radial wave functions $\phi_{P}(\hat{q})$ and $\phi_{V}(\hat{q})$ obtained as solutions of mass spectral equations [30-32] of pseudoscalar and vector quarkonia, respectively, that were obtained from 3D Salpeter equations being

$$
\begin{aligned}
\phi_{P, V}(1 S, \hat{q}) & =\frac{1}{\pi^{3 / 4}} \frac{1}{\beta_{P, V}^{3 / 2}} e^{-\frac{\hat{q}^{2}}{2 \beta_{P, V}^{2}}}, \\
\phi_{P, V}(2 S, \hat{q}) & =\sqrt{\frac{3}{2}} \frac{1}{\pi^{3 / 4}} \frac{1}{\beta_{P, V}^{3 / 2}}\left(1-\frac{2 \hat{q}^{2}}{3 \beta_{P, V}^{2}}\right) e^{-\frac{\hat{q}^{2}}{2 \beta_{P, V}^{2}}}, \\
\phi_{V}(1 D, \hat{q}) & =\sqrt{\frac{4}{15}} \frac{1}{\pi^{3 / 4}} \frac{1}{\beta_{V}^{7 / 2}} \hat{q}^{2} e^{-\frac{\hat{q}^{2}}{2 \beta_{V}^{2}}}, \\
\phi_{P, V}(3 S, \hat{q}) & =\sqrt{\frac{15}{8}} \frac{1}{\pi^{3 / 4}} \frac{1}{\beta_{P, V}^{3 / 2}}\left(1-\frac{4 \hat{q}^{2}}{3 \beta_{P, V}^{2}}+\frac{4 \hat{q}^{4}}{15 \beta_{P, V}^{4}}\right) e^{-\frac{\hat{q}^{2}}{2 \beta_{P, V}^{2}}},
\end{aligned}
$$

where $\beta_{P, V}$ [32] are the inverse range parameters.

These radial wave functions were recently used for calculations of some $M 1$ and $E 1$ transitions in [26]. We have made use of the 3D Salpeter equations in Eq. (6) that depend on the variable $\hat{q}^{2}$ that is Lorentz-invariant and is a four-scalar, whose validity extends over the entire 4D space, while also keeping contact with the surface $P . q=0$ (hadron rest frame). Now, our mass spectrum and the 3D wave functions $\phi(\hat{q})$ in Eq. (36) and (49) (please see [30]) were calculated from Salpeter equations in Eq. (6) in the rest frame of the hadron.

Here, $\hat{q}^{\prime 2}$ is expressed as [26]

$\hat{q}^{\prime 2}=\hat{q}^{2}+2 \hat{m}_{2} \frac{\left(M^{2}-M^{\prime 2}\right)}{2 M}|\hat{q}|+\hat{m}_{2}^{2} \frac{\left(M^{2}-M^{\prime 2}\right)^{2}}{4 M^{2}}$

where $|\hat{q}|$ is the length of the 3D vector $\hat{q}$, defined as $|\hat{q}|=\sqrt{\hat{q}^{2}}=\sqrt{q^{2}-(q \cdot P)^{2} / P^{2}}$, and is a Lorentz-invariant variable Ref. [26].

The Bethe-Salpeter normalizers $N_{P}$ and $N_{V}$ are obtained through the current conservation condition,

$$
\begin{aligned}
2 i P_{\mu}= & \int \frac{d^{4} q}{(2 \pi)^{4}} \operatorname{Tr}\left[\bar{\psi}(P, q)\left[\frac{\partial}{\partial P_{\mu}} S_{F}^{-1}\left(p_{1}\right)\right]\right. \\
& \left.\times \psi(P, q) S_{F}^{-1}\left(-p_{2}\right)\right]+(1 \leftrightarrow 2) .
\end{aligned}
$$

To evaluate $M_{\mathrm{fi}}$, we need the ++ and -- components of 3D BS wave functions of pseudoscalar and vector mesons and the corresponding adjoint wave functions of the pseudoscalar meson, which are given in Eqs. (A2)-(A6) of the Appendix. For E1 transitions, we can similarly construct the ++ and -- components of the axial meson wave functions.

The transition amplitude $M_{\mathrm{fi}}$ is expressed as

$$
M_{\mathrm{fi}}=F_{P V} \epsilon_{\mu \nu \alpha \beta} P_{\mu} \epsilon_{\nu}^{\lambda^{\prime}} \epsilon_{\alpha}^{\lambda} P_{\beta}^{\prime},
$$

where the antisymmetric tensor $\epsilon_{\mu \nu \alpha \beta}$ ensures its gauge invariance. Here, $F_{P V}$ is the transition form factor for $P \rightarrow V \gamma$, with the expression 
TABLE I. Masss spectra of ground and excited states of axial $\left(1^{+-}\right)$, pseudoscalar $\left(0^{-+}\right)$, and vector $\left(1^{--}\right)$ quarkonia (in $\mathrm{GeV}$ ) in BSE-CIA used in the transitions studied in this work, along with data and results of other models.

\begin{tabular}{lccccc}
\hline \hline & BSE-CIA[28,32] & Experiment[12] & BSE & Ref. [42-45] & RQM \\
\hline$M_{h_{c}\left(1 P_{1}\right)}$ & 3.535 & $3.525 \pm 0.00001$ & $3.5244[35]$ & $3.5059[42]$ & $3.525[46]$ \\
$M_{h_{c}\left(2 P_{1}\right)}$ & 3.743 & $3.888 \pm 0.0025$ & $3.9358[35]$ & & $3.927[46]$ \\
$M_{\eta_{c}\left(1 S_{0}\right)}$ & 3.0004 & $2.9839 \pm 0.0005$ & & $3.292[43]$ & $2.981[47]$ \\
$M_{\eta_{c}\left(2 S_{0}\right)}$ & 3.5934 & $3.6376 \pm 0.0012$ & & $4.240[43]$ & $3.635[47]$ \\
$M_{D\left(2 S_{0}\right)}$ & 2.5288 & & & $2.5235[44]$ & $2.581[47]$ \\
$M_{D^{*}\left(1 S_{0}\right)}$ & 2.0221 & $2.010 \pm 0.00005$ & & $2.0104[44]$ & $2.010[47]$ \\
$M_{D_{s}\left(2 S_{0}\right)}$ & 2.6358 & & $2.6333[44]$ & $2.6888[47]$ \\
$M_{D_{s}^{*}\left(1 S_{0}\right)}$ & 2.1153 & $2.1122 \pm 0.0004$ & & $2.1123[44]$ & $2.111[47]$ \\
$M_{J / \Psi(1 S)}$ & 3.0970 & $3.0969 \pm 0.0000025$ & & $3.099[42]$ & $3.096[48]$ \\
$M_{B_{c}^{*}\left(1 S_{1}\right)}$ & 6.3514 & & $6.321 \pm 0.020[45]$ & $6.332[48]$ \\
$M_{B_{c}\left(2 S_{0}\right)}$ & 6.7241 & & $6.960 \pm 0.080[45]$ & $6.835[48]$ \\
\hline \hline
\end{tabular}

$$
\begin{aligned}
F_{P V} & =F_{P V}^{1}+F_{P V}^{2}=N_{P} N_{V} \frac{1}{M^{2}} \int \frac{d^{3} \hat{q}}{(2 \pi)^{3}} \frac{\phi_{P}(\hat{q}) \phi_{V}\left(\hat{q}^{\prime}\right)}{16 \omega_{1} \omega_{2} \omega_{1}^{\prime} \omega_{2}^{\prime}} \\
& \times\left[e_{q}\left[T R_{1}\right]+e_{Q}\left[T R_{2}\right]\right],
\end{aligned}
$$

where the expressions for $F_{P V}^{1}$ and $F_{P V}^{2}$ are given in Eqs. (A6)-(A7) in the Appendix, and correspond to contributions from the two diagrams, which is a more accurate treatment. Here $\left[T R_{1}\right]$, and $\left[T R_{2}\right]$ involve trace over gamma matrices. The above expression corresponds to $F_{P V}\left(k^{2}=0\right)$, which corresponds to emission of a real photon.

The kinematical relation connecting $\hat{q}^{\prime 2}$ with $\hat{q}^{2}$ is given in Eq. (37). To calculate the decay widths, we need to calculate the spin averaged amplitude square $\left|\bar{M}_{\mathrm{fi}}\right|^{2}$, where $\left|\bar{M}_{\mathrm{fi}}\right|^{2}=\sum_{\lambda, \lambda^{\prime}}\left|M_{\mathrm{fi}}\right|^{2}$, where we sum over the final polarization states $\lambda$ of the $V$ meson and $\lambda^{\prime}$ of the photon. We make use of the normalizations $\Sigma_{\lambda} \epsilon_{\mu}^{\lambda} \epsilon_{\nu}^{\lambda}=\frac{1}{3}\left(\delta_{\mu \nu}+\frac{P_{\mu} P_{\nu}}{M^{2}}\right)$ for the vector meson and $\Sigma_{\lambda^{\prime}} \epsilon_{\mu}^{\lambda^{\prime}} \epsilon_{\nu}^{\lambda^{\prime}}=\delta_{\mu \nu}$ for the emitted photon, with $M_{\text {fi }}$ taken from Eq. (39). Thus, we write

$$
\left|\overline{M_{\mathrm{fi}}}\right|^{2}=-2 e^{2}\left[M^{2} M^{\prime 2}-\left(P . P^{\prime}\right)^{2}\right]\left|F_{P V}\right|^{2} .
$$

In the above equation, we evaluate $P \cdot P^{\prime}=-M E^{\prime}$ in the rest frame of the initial vector meson, where $E^{\prime}=$
$\sqrt{\vec{P}^{\prime 2}+M^{\prime 2}}$ is the energy of the final pseudoscalar meson, giving $P . P^{\prime}=-\left(\frac{M^{2}+M^{\prime 2}}{2}\right)$. Thus, $\left|\bar{M}_{\mathrm{fi}}\right|^{2}$ can be expressed as

$$
\left|\bar{M}_{\mathrm{fi}}\right|^{2}=e^{2} \frac{\left(M^{2}-M^{\prime 2}\right)^{2}}{2}\left|F_{P V}\right|^{2} .
$$

The decay width of the process $(P \rightarrow V \gamma)$ in the rest frame of the initial meson is expressed as

$$
\Gamma_{P \rightarrow V \gamma}=\frac{\left|\bar{M}_{\mathrm{fi}}\right|^{2}}{8 \pi M^{2}}\left|\vec{P}^{\prime}\right|
$$

where we make use of the fact that the modulus of the momentum of the emitted vector meson can be expressed in terms of masses of particles as $\left|\overrightarrow{P^{\prime}}\right|=|\vec{k}|=\omega_{k}=$ $\frac{1}{2 M}\left(M^{2}-M^{\prime 2}\right)$, where $\omega_{k}$ is the kinematically allowed energy of the emitted photon. Thus $\Gamma$ in turn can be expressed as

$$
\Gamma=\alpha_{e . m .}\left|F_{P V}\right|^{2} \omega_{k}^{3}
$$

The $M 1$ decay widths for the processes studied are given in Table II, for the meson masses given in Table I, that were evaluated in our previous works $[28,32]$.

TABLE II. Radiative decay widths of heavy-light mesons (in $\mathrm{keV}$.) for $M 1$ transitions in BSE, along with experimental data and results of other models.

\begin{tabular}{lcccccc}
\hline \hline & $\beta$ & BSE-CIA & Experiment & RQM & PM & RQM \\
\hline$\Gamma_{\eta_{c}\left(2 S_{0}\right) \rightarrow J / \Psi\left(1 S_{1}\right) \gamma}$ & 0.200 & 0.476 & $<158_{-40.6}^{+44.8}[12]$ & $0.700[48]$ & $2.29[49]$ & $5.6[50]$ \\
$\Gamma_{D\left(2 S_{0}\right) \rightarrow D^{*}\left(1 S_{1}\right) \gamma}$ & 0.180 & 12.157 & & & $8.594[51]$ & \\
$\Gamma_{D_{s}\left(2 S_{0}\right) \rightarrow D_{s}^{*}\left(1 S_{1}\right) \gamma}$ & 0.185 & 7.602 & & & & \\
$\Gamma_{B_{c}\left(2 S_{0}\right) \rightarrow B_{c}^{*}\left(1 S_{1}\right) \gamma}$ & 0.460 & 0.1028 & & $0.096[52]$ & $0.093[53]$ & $0.488[48]$ \\
\hline \hline
\end{tabular}


We now calculate the radiative decay widths for the process $A^{-} \rightarrow P+\gamma$ in the next section.

\section{RADIATIVE DECAYS OF HEAVY-LIGHT QUARKONIA THROUGH $\boldsymbol{A}^{-} \rightarrow \boldsymbol{P} \gamma$}

The $E 1$ transitions are characterized by $|\Delta L|=1$. Thus in these transitions, there is change in parity between the initial and final hadronic states. The scattering amplitude of the decay process $A^{-} \rightarrow P \gamma$ corresponding to diagram 1 can be written as

$$
\begin{aligned}
M_{\mathrm{fi}}^{1} & =-i e \int \frac{d^{3} \hat{q}}{(2 \pi)^{3}} \frac{1}{M^{2}} \operatorname{Tr}\left[\alpha_{1} P \bar{\psi}_{P}^{++}\left(\hat{q}^{\prime}\right) \epsilon^{\prime} \psi_{A}^{++}(\hat{q})\right. \\
& +\alpha_{2} P \bar{\psi}_{P}^{++}\left(\hat{q}^{\prime}\right) \epsilon^{\prime} \psi_{A}^{--}(\hat{q}) \\
& \left.+\alpha_{3} P \bar{\psi}_{P}^{--}\left(\hat{q}^{\prime}\right) \epsilon^{\prime} \psi_{A}^{++}(\hat{q})+\alpha_{4} P \bar{\psi}_{P}^{--}\left(\hat{q}^{\prime}\right) \epsilon \psi_{A}^{--}(\hat{q})\right] .
\end{aligned}
$$

Here, the results of contour integrals over $d \sigma$ are given as $\alpha_{1}, \ldots, \alpha_{4}$ in Eq. (29). Similarly the amplitude for diagram 2 can be written as

$$
\begin{aligned}
M_{\mathrm{fi}}^{2} & =-e_{Q} \int \frac{d^{3} \hat{q}}{(2 \pi)^{3}} \frac{1}{M^{2}} \operatorname{Tr}\left[\alpha_{1}^{\prime} \bar{\psi}_{P}^{++}\left(\hat{q}^{\prime}\right) P \psi_{A}^{++}(\hat{q}){\boldsymbol{\epsilon}^{\prime}}^{\prime}\right. \\
& +\alpha_{2}^{\prime} \bar{\psi}_{P}^{++}\left(\hat{q}^{\prime}\right) P \psi_{A}^{--}(\hat{q}) \hat{\epsilon}^{\prime} \\
& \left.+\alpha_{3}^{\prime} \bar{\psi}_{P}^{--}\left(\hat{q}^{\prime}\right) P \psi_{A}^{++}(\hat{q}) \hat{\boldsymbol{\epsilon}}^{\prime}+\alpha_{4}^{\prime} \bar{\psi}_{P}^{--}\left(\hat{q}^{\prime}\right) P \psi_{A}^{--}(\hat{q}) \boldsymbol{\epsilon}^{\prime}\right]
\end{aligned}
$$

And, the results of contour integrals over $d \sigma$ are given as $\alpha_{1}^{\prime}, \ldots, \alpha_{4}^{\prime}$ in Eq. (31). After the 3D reduction of the 4D BS wave function of $A^{-}$axial meson under CIA, we express the 3D BS wave function with dimensionality $M$ as

$$
\begin{aligned}
\psi_{A^{-}}(\hat{q})= & \gamma_{5}(\epsilon \cdot \hat{q})\left[g_{1}(\hat{q})+i \frac{P}{M} g_{2}(\hat{q})-i \frac{\hat{\phi}}{M} g_{3}(\hat{q})\right. \\
& \left.+2 \frac{P \hat{q}}{M^{2}} g_{4}(\hat{q})\right] .
\end{aligned}
$$

Making use of the fact that the leading order Dirac structures in an axial meson BS wave function in accordance with the power counting rule proposed in $[33,38,39]$ are $\gamma_{5} \epsilon . \hat{q}$, and $i \gamma_{5} \epsilon . \hat{q} \frac{P}{M}$, and making use of [31], we express the 3D axial meson BS wave function of dimension $M$ as

$$
\psi_{A^{-}}(\hat{q})=\gamma_{5}(\epsilon . \hat{q})\left[1+i \frac{P}{M}\right] \phi_{A^{-}}(\hat{q}),
$$

where $\phi_{A^{-}}(\hat{q})$ is the spatial part of this wave function, whose analytic forms obtained by power series solutions of 3D mass spectral equations [derived from 3D Salpeter equations in Eq. (6)], in the variable $\hat{q}$ (which is in fact $|\hat{q}|$ ) for a $P$-wave meson in its own rest frame, calculated in [31] are

$$
\begin{aligned}
& \phi_{A}(1 P, \hat{q})=\sqrt{\frac{2}{3}} \frac{1}{\pi^{3 / 4}} \frac{1}{\beta_{A}^{5 / 2}} \hat{q} e^{-\frac{\hat{q}^{2}}{2 \beta_{A}^{2}}}, \\
& \phi_{A}(2 P, \hat{q})=\sqrt{\frac{5}{3}} \frac{1}{\pi^{3 / 4}} \frac{1}{\beta_{A}^{5 / 2}} \hat{q}\left(1-\frac{2 \hat{q}^{2}}{5 \beta_{A}^{2}}\right) e^{-\frac{\hat{q}^{2}}{2 \beta_{A}^{2}}}, \\
& \phi_{A}(3 P, \hat{q})=\sqrt{\frac{35}{12}} \frac{1}{\pi^{3 / 4}} \frac{1}{\beta_{A}^{5 / 2}} \hat{q}\left(1-\frac{4 \hat{q}^{2}}{5 \beta_{A}^{2}}+\frac{4 \hat{q}^{4}}{35 \beta_{A}^{4}}\right) e^{-\frac{\hat{q}^{2}}{2 \beta_{A}^{2}}}, \\
& \phi_{A}(4 P, \hat{q})=\sqrt{\frac{35}{8}} \frac{1}{\pi^{3 / 4}} \frac{1}{\beta_{A}^{5 / 2}} \hat{q}\left(1-\frac{6 \hat{q}^{2}}{5 \beta_{A}^{2}}+\frac{12 \hat{q}^{4}}{35 \beta_{A}^{4}}-\frac{8 \hat{q}^{6}}{315 \beta_{A}^{6}}\right) e^{-\frac{\hat{q}^{2}}{2 \beta_{A}^{2}}} .
\end{aligned}
$$

It is to be noted that the wave functions in Eq. (49) involve even powers of $\hat{q}$, along with odd power $\hat{q}$, where $\hat{q}=|\hat{q}|$ is the length of the 3D vector $\hat{q}$, is expressed as $|\hat{q}|=\sqrt{q^{2}-(q \cdot P)^{2} / P^{2}}$, and is a Lorentz-invariant quantity [26], along with even powers of $\hat{q}$, such as $\hat{q}^{2}, \hat{q}^{4}, \ldots$ etc. which are again Lorentz invariant. And for S-wave mesons, the wave functions are only functions of even powers of $\hat{q}$. Thus when $P$-wave or $S$-wave mesons are in the final state, their wave functions after Lorentz transformation, would involve the variables $\left|\hat{q}^{\prime}\right|$, and/or even powers of $\hat{q}^{\prime}$. We express $\hat{q}^{\prime 2}$ in terms of $\hat{q}^{2}$ directly through Eq. (37), that connects $\hat{q}^{\prime 2}$ with $\hat{q}^{2}$, while odd power $\left|\hat{q}^{\prime}\right|$ is expressed as $\left|\hat{q}^{\prime}\right|=\sqrt{\hat{q}^{\prime 2}}$, where we again make use of Eq. (37). The BS normalizer of axial meson $N_{A^{-}}$can be obtained by solving the current conservation condition in Eq. (38).

We now obtain the ++ and -- components of the axial meson wave function with the corresponding adjoint wave functions as in the case of $P$ and $V$ mesons done earlier, and calculate expressions for,,++++++----++ , and - - -- terms that appear in the scattering amplitude in Eq. (46).

We then evaluate trace over the gamma matrices in Eq. (46). We make use of the fact that $\hat{q}^{\prime}=\hat{q}+\hat{m}_{2} \hat{P}^{\prime}$, where $\hat{P}^{\prime}=P^{\prime}-\frac{P^{\prime} . P}{P^{2}} P$. We combine various terms and further make use of the fact that for the initial axial meson $P . \epsilon^{\lambda}=0$. We can express $\hat{P}^{\prime} . \epsilon=P^{\prime} . \epsilon$. We can then express the invariant matrix element $M_{\mathrm{fi}}^{1}$ corresponding to diagram 1 as

$$
\begin{aligned}
M_{\mathrm{fi}}^{1}= & -i e N_{A} N_{P} \frac{1}{M} \int \frac{d^{3} \hat{q}}{(2 \pi)^{3}} \frac{\phi_{A}(\hat{q}) \phi_{P}\left(\hat{q}^{\prime}\right)}{16 \omega_{1} \omega_{2} \omega_{1}^{\prime} \omega_{2}^{\prime}} \\
& \times\left[\Theta_{1}\left(\epsilon^{\lambda^{\prime}} \cdot \epsilon^{\lambda}\right)+\Theta_{2}\left(\epsilon^{\lambda^{\prime}} \cdot P\right)\left(\epsilon^{\lambda} \cdot P^{\prime}\right)+\Theta_{3}\left(\epsilon^{\lambda^{\prime}} \cdot P^{\prime}\right)\left(\epsilon^{\lambda} \cdot P^{\prime}\right)\right],
\end{aligned}
$$

Similarly for diagram 2, we write the invariant matrix element $M_{\mathrm{fi}}^{2}$ as 


$$
\begin{aligned}
M_{\mathrm{fi}}^{2}= & -i e N_{A} N_{P} \frac{1}{M} \int \frac{d^{3} \hat{q}}{(2 \pi)^{3}} \frac{\phi_{A}(\hat{q}) \phi_{P}\left(\hat{q}^{\prime}\right)}{16 \omega_{1} \omega_{2} \omega_{1}^{\prime} \omega_{2}^{\prime}} \\
& \times\left[\Theta_{1}^{\prime}\left(\epsilon^{\lambda^{\prime}} \cdot \epsilon^{\lambda}\right)+\Theta_{2}^{\prime}\left(\epsilon^{\lambda^{\prime}} \cdot P\right)\left(\epsilon^{\lambda} \cdot P^{\prime}\right)+\Theta_{3}^{\prime}\left(\epsilon^{\lambda^{\prime}} \cdot P^{\prime}\right)\left(\epsilon^{\lambda} \cdot P^{\prime}\right)\right] .
\end{aligned}
$$

Total amplitude $M_{\mathrm{fi}}$ for the process can be expressed as the sum of amplitudes from the two diagrams $M_{\mathrm{fi}}=M_{\mathrm{fi}}^{1}+M_{\mathrm{fi}}^{2}$, where

$$
\begin{aligned}
M_{\mathrm{fi}} & =R_{1}\left(\epsilon^{\lambda^{\prime}} \cdot \epsilon^{\lambda}\right)+R_{2}\left(\epsilon^{\lambda^{\prime}} \cdot P\right)\left(\epsilon^{\lambda} \cdot P^{\prime}\right)+R_{3}\left(\epsilon^{\lambda} \cdot P^{\prime}\right)\left(\epsilon^{\lambda^{\prime}} \cdot P^{\prime}\right), \\
R_{1} & =-i e N_{A} N_{P} \frac{1}{M^{2}} \int \frac{d^{3} \hat{q}}{(2 \pi)^{3}} \frac{\phi_{P}\left(\hat{q}^{\prime}\right) \phi_{A}(\hat{q})}{16 \omega_{1} \omega_{2} \omega_{1}^{\prime} \omega_{2}^{\prime}}\left(\Theta_{1}+\Theta_{1}^{\prime}\right), \\
R_{2} & =-i e N_{A} N_{P} \frac{1}{M^{2}} \int \frac{d^{3} \hat{q}}{(2 \pi)^{3}} \frac{\phi_{P}\left(\hat{q}^{\prime}\right) \phi_{A}(\hat{q})}{16 \omega_{1} \omega_{2} \omega_{1}^{\prime} \omega_{2}^{\prime}}\left(\Theta_{2}+\Theta_{2}^{\prime}\right), \\
R_{3} & =-i e N_{A} N_{P} \frac{1}{M^{2}} \int \frac{d^{3} \hat{q}}{(2 \pi)^{3}} \frac{\phi_{P}\left(\hat{q}^{\prime}\right) \phi_{A}(\hat{q})}{16 \omega_{1} \omega_{2} \omega_{1}^{\prime} \omega_{2}^{\prime}}\left(\Theta_{3}+\Theta_{3}^{\prime}\right),
\end{aligned}
$$

where integrals over $d^{3} \hat{q}$ are performed over $\Theta_{i}$ and $\Theta_{i}^{\prime}$.

Thus $R_{1}, R_{2}$, and $R_{3}$ are the three form factors in the above equation. Now, with a change of variables, and making use of the electromagnetic gauge invariance, it can be shown that these three form factors appearing in $M_{\mathrm{fi}}$, are not independent, and we can express the invariant amplitude $M_{\mathrm{fi}}$ in terms of a single form factor.

To show this, we introduce two new external variables $\bar{P}$ and $k$, which are defined as

$$
\bar{P}=P+P^{\prime} ; k=P-P^{\prime}
$$

where $\bar{P}$ is the sum of momenta of the initial and emitted mesons and $k$ is the emitted photon momentum. Thus, we can express the initial and final meson momenta $P$ and $P^{\prime}$ in terms of new variables as $P=\frac{\bar{P}+k}{2}$ and $P^{\prime}=\frac{\bar{P}-k}{2}$. In terms of these new variables, we can express the amplitude $M_{\mathrm{fi}}$ as

$$
\begin{gathered}
M_{\mathrm{fi}}=S_{1}\left(\epsilon^{\prime} . \epsilon\right)+S_{2}(\bar{P} . \epsilon)\left(\bar{P} . \epsilon^{\prime}\right)+S_{3}(k . \epsilon)\left(\bar{P} . \epsilon^{\prime}\right), \\
S_{1}=R_{1} ; S_{2}=\frac{1}{4}\left(R_{2}+R_{3}\right) ; S_{3}=-\frac{1}{4}\left(R_{2}+R_{3}\right) .
\end{gathered}
$$

Now, the transversality property of the polarization vector of the axial meson P.e $=0$ leads to

$$
\epsilon_{\nu}(\bar{P}+k)_{\nu}=0 .
$$

We now introduce a new form factor $S^{\prime \prime}=-S_{2}+S_{3}$, in terms of which we can write $M_{\mathrm{fi}}$ as

$$
M_{\mathrm{fi}}=\epsilon_{\mu}^{\prime} \epsilon_{\nu}\left[S_{1} \delta_{\mu, \nu}+S_{2} \bar{P}_{\mu}(\bar{P}+k)_{\nu}+S^{\prime \prime} \bar{P}_{\mu} k_{\nu}\right] .
$$

Now due to Eq. (55), the term with $S_{2}$ vanishes. Thus, amplitude $M_{\mathrm{fi}}$ can be expressed as

$$
\begin{aligned}
M_{\mathrm{fi}} & =\epsilon_{\mu}^{\prime} \epsilon_{\nu} M_{\mu \nu} ; \\
M_{\mu \nu} & =S_{1} \delta_{\mu \nu}+S^{\prime \prime} \bar{P}_{\mu} k_{\nu} .
\end{aligned}
$$

Now, electromagnetic gauge invariance demands $k_{\mu} M_{\mu \nu}=0$. This leads to the relation

$$
S^{\prime \prime}=-\frac{S_{1}}{\bar{P} \cdot k}
$$

between the form factors, which is like an equation of constraint. Thus, due to the electromagnetic gauge invariance $S_{1}$ and $S^{\prime \prime}$ are no longer independent, and we can express the amplitude $M_{\mathrm{fi}}$ in terms of a single form factor $S_{1}$, whose expression is given in the next equations:

$$
\begin{aligned}
M_{\mathrm{fi}}= & S_{1}\left[\left(\epsilon^{\prime} \cdot \epsilon\right)-\frac{1}{\bar{P} \cdot k}\left(\bar{P} \cdot \epsilon^{\prime}\right)(k \cdot \epsilon)\right] ; \\
S_{1}= & -i e N_{A} N_{P} \frac{1}{M^{2}} \int \frac{d^{3} \hat{q}}{(2 \pi)^{3}} \frac{\phi_{P}\left(\hat{q}^{\prime}\right) \phi_{A}(\hat{q})}{16 \omega_{1} \omega_{2} \omega_{1}^{\prime} \omega_{2}^{\prime}} \\
& \times\left(\Theta_{1}\left(\hat{q}^{2}\right)+\Theta_{1}^{\prime}\left(\hat{q}^{2}\right)\right) .
\end{aligned}
$$

The expressions for $\Theta_{1}\left(\hat{q}^{2}\right)$ and $\Theta_{1}^{\prime}\left(\hat{q}^{2}\right)$ in the expression for $M_{\mathrm{fi}}$ above are

$$
\begin{aligned}
\Theta_{1}\left(\hat{q}^{2}\right)= & {\left[\left(-\alpha_{1} a_{5} b_{1}-\alpha_{2} a_{5} d_{1}-\alpha_{3} e_{5} b_{1}-\alpha_{4} e_{5} d_{1}\right)\left(-4 M^{2}\right) \hat{q}^{2}\right)+\left(-\alpha_{1} a_{8} b_{1}-\alpha_{2} a_{8} d_{1}-\alpha_{3} e_{8} b_{1}-\alpha_{4} e_{8} d_{1}\right) 4\left(P^{\prime} . P\right) \hat{q}^{2} } \\
& +\left(-\alpha_{1} a_{6} b_{2}-\alpha_{2} a_{6} d_{2}-\alpha_{3} e_{6} b_{2}-\alpha_{4} e_{6} d_{2}\right)\left(-4 M^{2}\right)\left(P^{\prime} . P\right) \hat{q}^{2}+\left(-\alpha_{1} a_{7} b_{2}-\alpha_{2} a_{7} d_{2}-\alpha_{3} e_{7} b_{2}-\alpha_{4} e_{7} d_{2}\right)\left(4 M^{2}\right) \hat{q}^{2} \\
& +\left(\alpha_{1} a_{9} b_{2}+\alpha_{2} a_{9} d_{2}+\alpha_{3} e_{9} b_{2}+\alpha_{4} e_{9} d_{2}\right)\left(-4 M^{2}\right)\left(P^{\prime} . P\right) \hat{q}^{2}+\left(-\alpha_{1} a_{1} b_{3}-\alpha_{2} a_{1} d_{3}-\alpha_{3} e_{1} b_{3}-\alpha_{4} e_{1} d_{3}\right)\left(4 M^{2}\right) \hat{q}^{2} \\
& +\left(-\alpha_{1} a_{4} b_{3}-\alpha_{2} a_{4} d_{3}-\alpha_{3} e_{4} b_{3}-\alpha_{4} e_{4} d_{3}\right)\left(4 M^{2}\right)\left(P^{\prime} . P\right) \hat{q}^{2} \\
& +\left(-\alpha_{1} a_{8} b_{3}-\alpha_{2} a_{8} d_{3}-\alpha_{3} e_{8} b_{3}-\alpha_{4} e_{8} d_{3}\right)\left(4 M^{2}\right)\left[\left(P^{\prime} . \hat{q}^{\prime}\right) \hat{q}^{2}+\left(P^{\prime} \cdot \hat{q}\right) \hat{q}^{2}\right] \\
& +\left(\alpha_{1} a_{2} b_{4}+\alpha_{2} a_{2} d_{4}+\alpha_{3} e_{2} b_{4}+\alpha_{4} e_{2} d_{4}\right)\left(4 M^{2}\right) \hat{q}^{2}+\left(\alpha_{1} a_{3} b_{4}+\alpha_{2} a_{3} d_{4}+\alpha_{3} e_{3} b_{4}+\alpha_{4} e_{3} d_{4}\right)\left(-4 P^{\prime} . P\right) \hat{q}^{2} \\
& +\left(\alpha_{1} a_{6} b_{4}+\alpha_{2} a_{6} d_{4}+\alpha_{3} e_{6} b_{4}+\alpha_{4} e_{6} d_{4}\right)\left(4 M^{2}\left(\hat{q}^{\prime} \cdot P^{\prime}\right) \hat{q}^{2}\right. \\
& \left.+\left(\alpha_{1} a_{9} b_{4}+\alpha_{2} a_{9} d_{4}+\alpha_{3} e_{9} b_{4}+\alpha_{4} e_{9} d_{4}\right)\left[4 M^{2} \hat{q}^{2}\left(P^{\prime} \cdot \hat{q}^{\prime}+P^{\prime} \cdot \hat{q}\right)\right]\right]
\end{aligned}
$$


and

$$
\begin{aligned}
\Theta_{1}^{\prime}\left(\hat{q}^{2}\right)= & {\left[\left(\alpha_{1}^{\prime} a_{5} b_{1}+\alpha_{2}^{\prime} a_{5} d_{1}+\alpha_{3}^{\prime} e_{5} b_{1}+\alpha_{4}^{\prime} e_{5} d_{1}\right)\left(-4 M^{2}\right) \hat{q}^{2}+\left(\alpha_{1}^{\prime} a_{8} b_{1}+\alpha_{2}^{\prime} a_{8} d_{1}+\alpha_{3}^{\prime} e_{8} b_{1}+\alpha_{4}^{\prime} e_{8} d_{1}\right) 4\left(P^{\prime} . P\right) \hat{q}^{2}\right.} \\
& +\left(\alpha_{1}^{\prime} a_{6} b_{2}+\alpha_{2}^{\prime} a_{6} d_{2}+\alpha_{3}^{\prime} e_{6} b_{2}+\alpha_{4}^{\prime} e_{6} d_{2}\right)\left(-4 M^{2}\right)\left(P^{\prime} . P\right) \hat{q}^{2}+\left(\alpha_{1}^{\prime} a_{7} b_{2}+\alpha_{2}^{\prime} a_{7} d_{2}+\alpha_{3}^{\prime} e_{7} b_{2}+\alpha_{4}^{\prime} e_{7} d_{2}\right)\left(4 M^{2}\right) \hat{q}^{2} \\
& +\left(\alpha_{1}^{\prime} a_{9} b_{2}+\alpha_{2}^{\prime} a_{9} d_{2}+\alpha_{3}^{\prime} e_{9} b_{2}+\alpha_{4}^{\prime} e_{9} d_{2}\right)\left(-12 M^{2}\right)\left(P^{\prime} . P\right) \hat{q}^{2}+\left(\alpha_{1}^{\prime} a_{1} b_{3}+\alpha_{2}^{\prime} a_{1} d_{3}+\alpha_{3}^{\prime} e_{1} b_{3}+\alpha_{4}^{\prime} e_{1} d_{3}\right)\left(-4 M^{2}\right) \hat{q}^{2} \\
& +\left(\alpha_{1}^{\prime} a_{4} b_{3}+\alpha_{2}^{\prime} a_{4} d_{3}+\alpha_{3}^{\prime} e_{4} b_{3}+\alpha_{4}^{\prime} e_{4} d_{3}\right)\left(-4 M^{2}\right)\left(P^{\prime} . P\right) \hat{q}^{2}+\left(\alpha_{1}^{\prime} a_{8} b_{3}+\alpha_{2}^{\prime} a_{8} d_{3}+\alpha_{3}^{\prime} e_{8} b_{3}+\alpha_{4}^{\prime} e_{8} d_{3}\right)\left(-4 M^{2}\right)\left(P^{\prime} . \hat{q}\right) \hat{q}^{2} \\
& +\left(\alpha_{1}^{\prime} a_{2} b_{4}+\alpha_{2}^{\prime} a_{2} d_{4}+\alpha_{3}^{\prime} e_{2} b_{4}+\alpha_{4}^{\prime} e_{2} d_{4}\right)\left(-4 M^{2}\right) \hat{q}^{2}+\left(\alpha_{1}^{\prime} a_{3} b_{4}+\alpha_{2}^{\prime} a_{3} d_{4}+\alpha_{3}^{\prime} e_{3} b_{4}+\alpha_{4}^{\prime} e_{3} d_{4}\right) 4\left(P^{\prime} . P\right) \hat{q}^{2} \\
& +\left(\alpha_{1}^{\prime} a_{6} b_{4}+\alpha_{2}^{\prime} a_{6} d_{4}+\alpha_{3}^{\prime} e_{6} b_{4}+\alpha_{4}^{\prime} e_{6} d_{4}\right)\left(-4 M^{2}\right)\left[\left(\hat{q}^{\prime} \cdot P^{\prime}\right) \hat{q}^{2}+\left(\hat{q} \cdot P^{\prime}\right) \hat{q}^{2}\right] \\
& \left.+\left(\alpha_{1}^{\prime} a_{9} b_{4}+\alpha_{2}^{\prime} a_{9} d_{4}+\alpha_{3}^{\prime} e_{9} b_{4}+\alpha_{4}^{\prime} e_{9} d_{4}\right)\left(4 M^{2}\right)\left(P^{\prime} . \hat{q}\right) \hat{q}^{2}\right]
\end{aligned}
$$

which is expressible in terms of dot products of various momenta, $\left(P^{\prime} . P\right)$ with expression in Eqs. (14), while $P^{\prime} . \hat{q}$, and $P^{\prime} \cdot \hat{q}^{\prime}$ can be expressed as

$$
\begin{aligned}
P^{\prime} . \hat{q} & =\frac{M^{2}-M^{\prime 2}}{2 M}|\hat{q}|, \\
P^{\prime} \cdot \hat{q}^{\prime} & =\frac{M^{2}-M^{\prime 2}}{2 M}|\hat{q}|+\hat{m}_{1} M^{\prime 2}+\frac{\left(M^{2}+M^{\prime 2}\right)^{2}}{4 M^{2}} .
\end{aligned}
$$

Further, $\alpha_{1}, \ldots, \alpha_{4}$, and $\alpha_{1}^{\prime}, \ldots, \alpha_{4}^{\prime}$ are the results of contour integrals over the poles of the propagators in Eqs. (29) and (31) respectively, and the expressions for coefficients $a_{i}, b_{i}, d_{i}$, and $e_{i}$ are given in Eq. (A9) of the Appendix.

Now, to calculate the decay widths, we need to calculate the spin averaged amplitude modulus square $\left|\bar{M}_{\mathrm{fi}}\right|^{2}$, where $\left|\bar{M}_{\mathrm{fi}}\right|^{2}=\frac{1}{2 j+1} \sum_{\lambda, \lambda^{\prime}}\left|M_{\mathrm{fi}}\right|^{2}$, where we average over the initial polarization states $\lambda$ of the $A$ meson, and sum over the final polarization $\lambda^{\prime}$ of the photon. We make use of the normalizations $\Sigma_{\lambda} \epsilon_{\mu}^{\lambda} \epsilon_{\nu}^{\lambda}=\frac{1}{3}\left(\delta_{\mu \nu}+\frac{P_{\mu} P_{\nu}}{M^{2}}\right)$ for the vector meson, and $\Sigma_{\lambda^{\prime}} \epsilon_{\mu}^{\lambda^{\prime}} \epsilon_{\nu}^{\lambda^{\prime}}=\delta_{\mu \nu}$, for the emitted photon, with $M_{\mathrm{fi}}$ taken from the previous equation, and we get $\sum_{\lambda^{\prime}} \sum_{\lambda}\left|\epsilon^{\lambda^{\prime}} \cdot \epsilon^{\lambda}\right|^{2}=1$.

Making use of the kinematical relations,

$$
\begin{aligned}
\bar{P} . k & =-M^{2}+M^{\prime 2}, \\
P . k & =\frac{-M^{2}+M^{\prime 2}}{2}, \\
\bar{P}^{2} & =-2\left(M^{2}+M^{\prime 2}\right), \\
\bar{P} . P & =\frac{-3 M^{2}-M^{\prime 2}}{2},
\end{aligned}
$$

the spin-averaged amplitude modulus square of the process can be written as

$$
\left|\bar{M}_{\mathrm{fi}}\right|^{2}=\frac{1}{3}\left|S_{1}\right|^{2}\left[1-\frac{M^{\prime 2}}{3 M^{2}}\right]
$$

We can write the decay width as

$$
\Gamma_{A \rightarrow P \gamma}=\frac{\left|\bar{M}_{\mathrm{fi}}\right|^{2}}{8 \pi M^{2}}\left|\overrightarrow{P^{\prime}}\right|,
$$

where we make use of the fact that the modulus of the momentum of the final pseudoscalar meson can be expressed in terms of masses of particles as $\left|\vec{P}^{\prime}\right|=\frac{1}{2 M}\left(M^{2}-M^{\prime 2}\right)$.

\section{RADIATIVE DECAYS OF HEAVY-LIGHT QUARKONIA THROUGH $\boldsymbol{P} \rightarrow \boldsymbol{A}^{-} \gamma$}

We proceed to evaluate the process $P \rightarrow A^{-} \gamma$ using Fig. 1. Here, the initial pseudoscalar $\left(0^{-+}\right)$meson decays into an axial vector $\left(1^{+-}\right)$meson and a photon. We can then express the effective 3D form of an invariant matrix element $M_{\mathrm{fi}}^{1}$ corresponding to diagram 1 as

$$
\begin{aligned}
M_{\mathrm{fi}}^{1}= & -i e \int \frac{d^{3} \hat{q}}{(2 \pi)^{3}} \frac{1}{M^{2}} \operatorname{Tr}\left[\alpha_{1} P \bar{\psi}_{A}^{++}\left(\hat{q}^{\prime}\right) \epsilon^{\prime} \psi_{P}^{++}(\hat{q})\right. \\
& +\alpha_{2} P \bar{\psi}_{A}^{++}\left(\hat{q}^{\prime}\right) \epsilon^{\prime} \psi_{P}^{--}(\hat{q}) \\
& \left.+\alpha_{3} P \bar{\psi}_{A}^{--}\left(\hat{q}^{\prime}\right) \epsilon^{\prime} \psi_{P}^{++}(\hat{q})+\alpha_{4} P \bar{\psi}_{A}^{--}\left(\hat{q}^{\prime}\right) \epsilon^{\prime} \psi_{P}^{--}(\hat{q})\right] .
\end{aligned}
$$

Similarly the amplitude for diagram 2 can be written as

$$
\begin{aligned}
M_{\mathrm{fi}}^{2}= & -e_{Q} \int \frac{d^{3} \hat{q}}{(2 \pi)^{3}} \frac{1}{M^{2}} \operatorname{Tr}\left[\alpha_{1}^{\prime} \bar{\psi}_{A}^{++}\left(\hat{q}^{\prime}\right) P \psi_{P}^{++}(\hat{q}) \hat{\epsilon}^{\prime}\right. \\
& +\alpha_{2}^{\prime} \bar{\psi}_{A}^{++}\left(\hat{q}^{\prime}\right) P \psi_{P}^{--}(\hat{q}) \boldsymbol{\epsilon}^{\prime} \\
& \left.+\alpha_{3}^{\prime} \bar{\psi}_{A}^{--}\left(\hat{q}^{\prime}\right) P \psi_{P}^{++}(\hat{q}) \hat{\epsilon}^{\prime}+\alpha_{4}^{\prime} \bar{\psi}_{A}^{--}\left(\hat{q}^{\prime}\right) P \psi_{P}^{--}(\hat{q}) \hat{\epsilon}^{\prime}\right],
\end{aligned}
$$

$\alpha_{1}, \ldots, \alpha_{4}$ and $\alpha_{1}^{\prime}, \ldots, \alpha_{4}^{\prime}$ in Eq. (29), and Eqs. (31) are the results of pole integrations over $M d \sigma$. 
Taking trace over the gamma matrices in the previous equations, we get the invariant matrix element $M_{\mathrm{fi}}^{1}$ for diagram 1 as

$$
\begin{aligned}
M_{\mathrm{fi}}^{1}= & -i e N_{A} N_{P} \frac{1}{M^{2}} \int \frac{d^{3} \hat{q}}{(2 \pi)^{3}} \frac{\phi_{A}\left(\hat{q}^{\prime}\right) \phi_{P}(\hat{q})}{16 \omega_{1} \omega_{2} \omega_{1}^{\prime} \omega_{2}^{\prime}} \\
& {\left[\Delta_{1}\left(\epsilon^{\lambda^{\prime}} \cdot \epsilon^{\lambda}\right)+\Delta_{2}\left(\epsilon^{\lambda^{\prime}} \cdot P\right)\left(\epsilon^{\lambda} \cdot P\right)+\Delta_{3}\left(\epsilon^{\lambda^{\prime}} \cdot P^{\prime}\right)\left(\epsilon^{\lambda} \cdot P\right)\right] . }
\end{aligned}
$$

Similarly for diagram 2 , we write the invariant matrix element $M_{\mathrm{fi}}^{2}$ as

$$
\begin{aligned}
M_{\mathrm{fi}}^{2}= & -i e N_{A} N_{P} \frac{1}{M^{2}} \int \frac{d^{3} \hat{q}}{(2 \pi)^{3}} \frac{\phi_{A}\left(\hat{q}^{\prime}\right) \phi_{P}(\hat{q})}{16 \omega_{1} \omega_{2} \omega_{1}^{\prime} \omega_{2}^{\prime}} \\
& \times\left[\Delta_{1}^{\prime}\left(\epsilon^{\lambda^{\prime}} \cdot \epsilon^{\lambda}\right)+\Delta_{2}^{\prime}\left(\epsilon^{\lambda^{\prime}} \cdot P\right)\left(\epsilon^{\lambda} \cdot P\right)+\Delta_{3}^{\prime}\left(\epsilon^{\lambda^{\prime}} \cdot P^{\prime}\right)\left(\epsilon^{\lambda} \cdot P\right)\right],
\end{aligned}
$$

The total amplitude $M_{\mathrm{fi}}$ for the process can be expressed as the sum of amplitudes from the two diagrams $M_{\mathrm{fi}}=M_{\mathrm{fi}}^{1}+M_{\mathrm{fi}}^{2}$, where

$$
\begin{aligned}
M_{\mathrm{fi}} & =S_{1}^{\prime}\left(\epsilon^{\lambda^{\prime}} \cdot \epsilon^{\lambda}\right)+S_{2}^{\prime}\left(\epsilon^{\lambda} \cdot P\right)\left(\epsilon^{\lambda^{\prime}} \cdot P\right)+S_{3}^{\prime}\left(\epsilon^{\lambda} \cdot P\right)\left(\epsilon^{\lambda^{\prime}} \cdot P^{\prime}\right), \\
S_{1}^{\prime} & =-i e N_{P} N_{A} \int \frac{d^{3} \hat{q}}{(2 \pi)^{3}} \frac{\phi_{P}(\hat{q}) \phi_{A}\left(\hat{q}^{\prime}\right)}{16 \omega_{1} \omega_{2} \omega_{1}^{\prime} \omega_{2}^{\prime} M^{4} M^{\prime}}\left(\Delta_{1}\left(\hat{q}^{2}\right)+\Delta_{1}^{\prime}\left(\hat{q}^{2}\right)\right), \\
S_{2}^{\prime} & =-i e N_{P} N_{A} \int \frac{d^{3} \hat{q}}{(2 \pi)^{3}} \frac{\phi_{P}(\hat{q}) \phi_{A}\left(\hat{q}^{\prime}\right)}{16 \omega_{1} \omega_{2} \omega_{1}^{\prime} \omega_{2}^{\prime} M^{4} M^{\prime}}\left(\Delta_{2}\left(\hat{q}^{2}\right)+\Delta_{2}^{\prime}\left(\hat{q}^{2}\right)\right), \\
S_{3}^{\prime} & =-i e N_{P} N_{A} \int \frac{d^{3} \hat{q}}{(2 \pi)^{3}} \frac{\phi_{P}(\hat{q}) \phi_{A}\left(\hat{q}^{\prime}\right)}{16 \omega_{1} \omega_{2} \omega_{1}^{\prime} \omega_{2}^{\prime} M^{4} M^{\prime}}\left(\Delta_{3}\left(\hat{q}^{2}\right)+\Delta_{3}^{\prime}\left(\hat{q}^{2}\right)\right) .
\end{aligned}
$$

The structure of $M_{\mathrm{fi}}$ above is similar to [25]. To calculate the decay widths, we again need to calculate the spin averaged amplitude modulus square $\left|\bar{M}_{\mathrm{fi}}\right|^{2}$, where $\left|\bar{M}_{\mathrm{fi}}\right|^{2}=\sum_{\lambda, \lambda^{\prime}}\left|M_{\mathrm{fi}}\right|^{2}$, where we sum over the final polarization states $\lambda^{\prime}$ of the photon and $\lambda$ of the $V$ meson. Following a similar procedure as in $A^{-} \rightarrow P \gamma$, it is seen that the contributions to a spin averaged amplitude modulus square arises only from $S_{1}^{\prime}$, while the contributions from $S_{2}^{\prime}$ and $S_{3}^{\prime}$ vanish after doing the averaging over the polarization states.
The spin averaged amplitude modulus square gives

$$
\left|\bar{M}_{\mathrm{fi}}\right|^{2}=\left|S_{1}^{\prime}\right|^{2},
$$

for evaluating that which we need to evaluate the form factor $S_{1}$, for which we need to perform $\int \frac{d^{3} \hat{q}}{(2 \pi)^{3}}$ integration over $\left.\left(\Delta_{1}\left(\hat{q}^{2}\right)\right)+\Delta_{1}^{\prime}\left(\hat{q}^{2}\right)\right)$. The expression for $\left.\Delta_{1}\left(\hat{q}^{2}\right)\right)$ that arises from diagram 1 is

$$
\begin{aligned}
\Delta_{1}\left(\hat{q}^{2}\right)= & {\left[4 M^{2} \hat{q}^{2}\left(\alpha_{1} b_{4}^{\prime} a_{1}^{\prime}+\alpha_{2} b_{4}^{\prime} d_{1}^{\prime}+\alpha_{3} e_{4}^{\prime} a_{1}^{\prime}+\alpha_{4} e_{4}^{\prime} d_{1}^{\prime}\right)+4\left(P^{\prime} . P\right) \hat{q}^{2}\left(\alpha_{1} b_{8}^{\prime} a_{1}^{\prime}+\alpha_{2} b_{8}^{\prime} d_{1}^{\prime}+\alpha_{3} e_{8}^{\prime} a_{1}^{\prime}+\alpha_{4} e_{8}^{\prime} d_{1}^{\prime}\right)\right.} \\
& +4 M^{2}\left(P^{\prime} . P\right) \hat{q}^{2}\left(\alpha_{1} b_{6}^{\prime} a_{2}^{\prime}+\alpha_{2} b_{6}^{\prime} d_{2}^{\prime}+\alpha_{3} e_{6}^{\prime} a_{2}^{\prime}+\alpha_{4} e_{6}^{\prime} d_{2}^{\prime}\right) \\
& +4 M^{2} \hat{q}^{2}\left(\alpha_{1} b_{7}^{\prime} a_{2}^{\prime}+\alpha_{2} b_{7}^{\prime} d_{2}^{\prime}+\alpha_{3} e_{7}^{\prime} a_{2}^{\prime}+\alpha_{4} e_{7}^{\prime} d_{2}^{\prime}\right)+-4 M^{2}\left(P^{\prime} . \hat{q}\right) \hat{q}^{2}\left(\alpha_{1} b_{6}^{\prime} a_{4}^{\prime}+\alpha_{2} b_{6}^{\prime} d_{4}^{\prime}+\alpha_{3} e_{6}^{\prime} a_{4}^{\prime}+\alpha_{4} e_{6}^{\prime} d_{4}^{\prime}\right) \\
& +4 M^{2} \hat{q}^{2}\left(P . P^{\prime}\right)\left(\alpha_{1} b_{9}^{\prime} a_{2}^{\prime}+\alpha_{2} b_{9}^{\prime} d_{2}^{\prime}+\alpha_{3} e_{9}^{\prime} a_{2}^{\prime}+\alpha_{4} e_{9}^{\prime} d_{2}^{\prime}\right) \\
& +4 M^{2} \hat{q}^{2}\left(\alpha_{1} b_{1}^{\prime} a_{3}^{\prime}+\alpha_{2} b_{1}^{\prime} d_{3}^{\prime}+\alpha_{3} e_{1}^{\prime} a_{3}^{\prime}+\alpha_{4} e_{1}^{\prime} d_{3}^{\prime}\right)+4 M^{2}\left(P^{\prime} . P\right) \hat{q}^{2}\left(\alpha_{1} b_{5}^{\prime} a_{3}^{\prime}+\alpha_{2} b_{5}^{\prime} d_{3}^{\prime}+\alpha_{3} e_{5}^{\prime} a_{3}^{\prime}+\alpha_{4} e_{5}^{\prime} d_{3}^{\prime}\right) \\
& +4 M^{2}\left(P^{\prime} \cdot \hat{q}^{\prime}\right) \hat{q}^{2}\left(\alpha_{1} b_{8}^{\prime} a_{3}^{\prime}+\alpha_{2} b_{8}^{\prime} d_{3}^{\prime}+\alpha_{3} e_{8}^{\prime} a_{3}^{\prime}+\alpha_{4} e_{8}^{\prime} d_{3}^{\prime}\right)+4 M^{2} \hat{q}^{2}\left(\alpha_{1} b_{2}^{\prime} a_{4}^{\prime}+\alpha_{2} b_{2}^{\prime} d_{4}^{\prime}+\alpha_{3} e_{2}^{\prime} a_{4}^{\prime}+\alpha_{4} e_{2}^{\prime} d_{4}^{\prime}\right) \\
& \left.-4\left(P^{\prime} . P\right) \hat{q}^{2}\left(\alpha_{1} b_{3}^{\prime} a_{4}^{\prime}+\alpha_{2} b_{3}^{\prime} d_{4}^{\prime}+\alpha_{3} e_{3}^{\prime} a_{4}^{\prime}+\alpha_{4} e_{4}^{\prime} d_{4}^{\prime}\right)-4 M^{2}\left(P^{\prime} . \hat{q}^{\prime}\right) \hat{q}^{2}\left(\alpha_{1} b_{6}^{\prime} a_{4}^{\prime}+\alpha_{2} b_{6}^{\prime} d_{4}^{\prime}+\alpha_{3} e_{6}^{\prime} a_{4}^{\prime}+\alpha_{4} e_{6}^{\prime} d_{4}^{\prime}\right)\right],
\end{aligned}
$$

while the expression for $\Delta_{1}^{\prime}$ arising from for diagram 2 reads 


$$
\begin{aligned}
\Delta_{1}^{\prime}\left(\hat{q}^{2}\right)= & {\left[-\left(\alpha_{1}^{\prime} b_{6} a_{4}+\alpha_{2}^{\prime} b_{6}^{\prime} d_{4}^{\prime}+\alpha_{3}^{\prime} e_{6}^{\prime} a_{4}^{\prime}+\alpha_{4}^{\prime} e_{6}^{\prime} d_{4}^{\prime}\right)\left(P^{\prime} . \hat{q}\right)\left(4 M^{2}\right) \hat{q}^{2}+\left(\alpha_{1}^{\prime} b_{4}^{\prime} a_{1}^{\prime}+\alpha_{2}^{\prime} b_{4}^{\prime} d_{1}^{\prime}+\alpha_{3}^{\prime} e_{4}^{\prime} a_{1}^{\prime}+\alpha_{4}^{\prime} e_{4}^{\prime} d_{1}^{\prime}\right)\left(-4 M^{2}\right) \hat{q}^{2}\right.} \\
& +\left(\alpha_{1}^{\prime} b_{8}^{\prime} a_{1}^{\prime}+\alpha_{2}^{\prime} b_{8}^{\prime} d_{1}^{\prime}+\alpha_{3}^{\prime} e_{8}^{\prime} a_{1}^{\prime}+\alpha_{4}^{\prime} e_{8}^{\prime} d_{1}^{\prime}\right) 4\left(P^{\prime} . P\right) \hat{q}^{2}+\left(\alpha_{1}^{\prime} b_{6}^{\prime} a_{2}^{\prime}+\alpha_{2}^{\prime} b_{6}^{\prime} d_{2}^{\prime}+\alpha_{3}^{\prime} e_{6}^{\prime} a_{2}^{\prime}+\alpha_{4}^{\prime} e_{6}^{\prime} d_{2}^{\prime}\right) 8 M^{2}\left(P^{\prime} . P\right) \hat{q}^{2} \\
& +\left(\alpha_{1}^{\prime} b_{7}^{\prime} a_{2}^{\prime}+\alpha_{2}^{\prime} b_{7}^{\prime} d_{2}^{\prime}+\alpha_{3}^{\prime} e_{7}^{\prime} a_{2}^{\prime}+\alpha_{4}^{\prime} e_{7}^{\prime} d_{2}^{\prime}\right) 4 M^{2} \hat{q}^{2}+\left(\alpha_{1}^{\prime} b_{8}^{\prime} a_{3}^{\prime}+\alpha_{2}^{\prime} b_{8}^{\prime} d_{3}^{\prime}+\alpha_{3}^{\prime} e_{8}^{\prime} a_{3}^{\prime}+\alpha_{4}^{\prime} e_{8}^{\prime} d_{3}^{\prime}\right) 4 M^{2}\left(P^{\prime} . \hat{q}\right) \hat{q}^{2} \\
& +\left(\alpha_{1}^{\prime} b_{9}^{\prime} a_{2}^{\prime}+\alpha_{2}^{\prime} b_{9}^{\prime} d_{2}^{\prime}+\alpha_{3}^{\prime} e_{9}^{\prime} a_{2}^{\prime}+\alpha_{4}^{\prime} e_{9}^{\prime} d_{2}^{\prime}\right) 4 M^{2}\left(P^{\prime} . P\right) \hat{q}^{2}+\left(\alpha_{1}^{\prime} b_{1}^{\prime} a_{3}^{\prime}+\alpha_{2}^{\prime} b_{1}^{\prime} d_{3}^{\prime}+\alpha_{3}^{\prime} e_{1}^{\prime} a_{3}^{\prime}+\alpha_{4}^{\prime} e_{1}^{\prime} d_{3}^{\prime}\right) 4 M^{2} \hat{q}^{2} \\
& +\left(\alpha_{1}^{\prime} b_{5}^{\prime} a_{3}^{\prime}+\alpha_{2}^{\prime} b_{5}^{\prime} d_{3}^{\prime}+\alpha_{3}^{\prime} e_{5}^{\prime} a_{3}^{\prime}+\alpha_{4}^{\prime} e_{5}^{\prime} d_{3}^{\prime}\right) 4 M^{2}\left(P^{\prime} . P\right) \hat{q}^{2}+\left(\alpha_{1}^{\prime} b_{8}^{\prime} a_{3}^{\prime}+\alpha_{2}^{\prime} b_{8}^{\prime} d_{3}^{\prime}+\alpha_{3}^{\prime} e_{8}^{\prime} a_{3}^{\prime}+\alpha_{4}^{\prime} e_{8}^{\prime} d_{3}^{\prime}\right) 4 M^{2}\left(P^{\prime} . \hat{q}^{\prime}\right) \hat{q}^{2} \\
& +\left(\alpha_{1}^{\prime} b_{2}^{\prime} a_{4}^{\prime}+\alpha_{2}^{\prime} b_{2}^{\prime} d_{4}^{\prime}+\alpha_{3}^{\prime} e_{2}^{\prime} a_{4}^{\prime}+\alpha_{4}^{\prime} e_{2}^{\prime} d_{4}^{\prime}\right) 4 M^{2} \hat{q}^{2}+\left(\alpha_{1}^{\prime} b_{3}^{\prime} a_{4}^{\prime}+\alpha_{2}^{\prime} b_{3}^{\prime} d_{4}^{\prime}+\alpha_{3}^{\prime} e_{3}^{\prime} a_{4}^{\prime}+\alpha_{4}^{\prime} e_{3}^{\prime} d_{4}^{\prime}\right) 4\left(P^{\prime} . P\right) \hat{q}^{2} \\
& \left.+\left(\alpha_{1}^{\prime} b_{6}^{\prime} a_{4}^{\prime}+\alpha_{2}^{\prime} b_{6}^{\prime} d_{4}^{\prime}+\alpha_{3}^{\prime} e_{6}^{\prime} a_{4}^{\prime}+\alpha_{4}^{\prime} e_{6}^{\prime} d_{4}^{\prime}\right) 4 M^{2}\left(P^{\prime} . \hat{q}^{\prime}\right) \hat{q}^{2}+\left(\alpha_{1}^{\prime} b_{9}^{\prime} a_{4}^{\prime}+\alpha_{2}^{\prime} b_{9}^{\prime} d_{4}^{\prime}+\alpha_{3}^{\prime} e_{9}^{\prime} a_{4}^{\prime}+\alpha_{4}^{\prime} e_{9}^{\prime} d_{4}^{\prime}\right) 8 M^{2}\left(P^{\prime} . \hat{q}\right) \hat{q}^{2}\right],
\end{aligned}
$$

which is again expressible in terms of dot products of various momenta, $\left(P^{\prime} . P\right), P^{\prime} . \hat{q}$, and $P^{\prime} . \hat{q}^{\prime}$ with expressions in Eqs. (14) and (62) respectively, and $\alpha_{1}, \ldots, \alpha_{4}$ and $\alpha_{1}^{\prime}, \ldots, \alpha_{4}^{\prime}$ are the results of the contour integrations over the poles of the propagators in Eqs. (29) and (31). The coefficients $a_{i}^{\prime}, b^{\prime}{ }_{i}, d^{\prime}{ }_{i}, e^{\prime}{ }_{i}$ entering into $\Delta_{1}$, and $\Delta_{1}^{\prime}$ above are given in Eq. (A10) of the Appendix.

The decay widths $\Gamma$ for the process $P \rightarrow A^{-} \gamma$ are given by Eq. (65) with $P^{\prime}$ now the momentum of the emitted axial meson.

\section{RESULTS AND DISCUSSION}

The present work is an extension of our work in [26] to study of radiative $M 1$ decays $P \rightarrow V \gamma$ and $E 1$ decays $A^{-} \rightarrow$ $P \gamma$ and $P \rightarrow A^{-} \gamma$ of heavy-light quarkonia in the framework of a $4 \times 4$ BSE under covariant instantaneous ansatz, which is a Lorentz-invariant generalization of instantaneous approximation. In our recent work [26], we had studied the processes $V \rightarrow P \gamma, V \rightarrow S \gamma$, and $S \rightarrow V \gamma$. Such processes involve quark-triangle diagrams, involve two hardon-quark vertices, and are difficult to evaluate in BSE under CIA. We have made use of the generalized method of handling quark-triangle diagrams with two hadron-quark vertices in the framework of a $4 \times 4$ BSE by expressing the transition amplitude $M_{\mathrm{fi}}$ as a linear superposition of terms (shown in [26]) involving all possible combinations of ++ and -components of Salpeter wave functions of final and initial hadrons, through the terms,,++++----++-- , and --++ , with each of the four terms being associated with a coefficient $\alpha_{i}(i=1, \ldots, 4)$, which is the result of pole integration in the complex $\sigma$ plane. This superposition of all possible terms is a feature of relativistic frameworks.

Using this generalized expression for $M_{\mathrm{fi}}$, in Eqs. (28)(31), we have evaluated the decay widths for $M 1$ transitions ${ }^{1} S_{0} \rightarrow{ }^{3} S_{1}+\gamma$ involving the decays of the ground and excited states of the heavy-light mesons such as $\eta_{c}(2 S), B_{c}(2 S)$. We mention that as seen from Tables II and III, the decay rates of $M 1$ transitions are much weaker than the rates for $E 1$ transitions. But $M 1$ decay rates are interesting as they allow access to spin-singlet states, which are very difficult to produce. It can be seen that Table II for $M 1$ transitions shows only the upper limit of the experimental value of $\Gamma<158_{-40.6}^{+44.8} \mathrm{KeV}[12]$, which is the decay width for the transition $\eta_{c}(2 S) \rightarrow J / \Psi(1 S)+\gamma$. And the theoretical results are much below this upper limit. The experimental data for other transitions is not available yet.

In our previous work[26], we simplified the calculation by considering only the most leading Dirac structures in the wave functions of $P, V$, and $S$ mesons, that contribute the maximum to the calculation of all meson observables in accordance with our power counting rule as seen in our previous studies on leptonic decay constants and two photon decays of a meson [33,38]. However, in the present calculation, we consider the two leading order Dirac structures in the BS wave functions of $P, V$, and $A$ mesons given in Eqs. (35) and (48) in accordance with the power

TABLE III. Radiative decay widths of heavy-light mesons (in $\mathrm{keV}$ ) for $E 1$ transitions in BSE, along with experimental data and results of other models.

\begin{tabular}{lcccccc}
\hline \hline & $\beta$ & BSE-CIA & Experiment & [48] & PM & RQM \\
\hline$\Gamma_{h_{c}(1 P) \rightarrow \eta_{c}\left(1 S_{0}\right) \gamma}$ & 0.253 & 363.047 & $357 \pm 204[12]$ & $560[48]$ & $398 \pm 99[19]$ & $482[13]$ \\
$\Gamma_{h_{c}(2 P) \rightarrow \eta_{c}\left(2 S_{0}\right) \gamma}$ & 0.471 & 187.145 & & & $160[27]$ & $218[50]$ \\
$\Gamma_{h_{c}(2 P) \rightarrow \eta_{c}\left(1 S_{0}\right) \gamma}$ & 0.510 & 20.195 & & & $135[27]$ & $85[50]$ \\
$\Gamma_{\eta_{c}\left(2 S_{0}\right) \rightarrow h_{c}(1 P) \gamma}$ & 0.650 & 6.909 & & $6.2[48]$ & $49[50]$ & \\
\hline \hline
\end{tabular}


counting scheme we proposed in $[33,38]$, which makes this calculation more rigorous.

Now, for processes where a single hadron is involved, such as leptonic and two-photon decays of a hadron, it is possible to study partial contributions and relevance of different Dirac structures in hadronic BS wave functions of the hadron by employing a power counting rule $[33,38,39]$ we had proposed. In other works $[37,41]$ also, the contribution of separate Dirac structures were studied only for mass spectral studies and leptonic decays of hadrons where a single hadron is involved in the process.

However, for single photon radiative decay process such as $P \rightarrow V \gamma$, where two hadrons are involved, it is not possible to find partial contributions from individual Dirac structures appearing in hadronic BS wave functions, since, we notice that there is a complete mixing up of the Dirac structures appearing in the BS wave functions of the two different hadrons, and one can not cleanly disentangle their contributions. This is due to the fact that the amplitude, $M_{f i}$ given in Eqs. (28) and (30) involves terms like, $\operatorname{Tr}\left[\alpha_{1} P \bar{\psi}_{V}^{++}\left(\hat{q}^{\prime}\right) \boldsymbol{\epsilon}^{\prime} \psi_{P}^{++}(\hat{q})+\ldots ..\right]$, where, $\bar{\psi}_{V}^{++}\left(\hat{q}^{\prime}\right)$ involves the vector meson wave function, $\psi_{V}(\hat{q})$, which is expressible as a superposition of two leading order Dirac structures, $\gamma_{V_{1}}=i M \not \ell$ and $\gamma_{V_{2}}=\not \subset P$, as in Eq. (35), of which $\gamma_{V_{1}}$ is the more dominant structure, while $\psi_{P}^{++}(\hat{q})$ involves pseudoscalar meson wave function, $\psi_{P}(\hat{q})$, which is expressible in terms of two leading order Dirac structures, $\gamma_{P 1} \equiv M \gamma_{5}$, and $\gamma_{P_{2}} \equiv i P \gamma_{5}$, of which $\gamma_{P_{1}}$ was the more dominant structure.

Now, we write the total amplitude $M_{\mathrm{fi}}=M_{\mathrm{fi}}^{1}+M_{\mathrm{fi}}^{2}$ using Eqs. (28) and (30), each of which involves trace over the superposition of the mixtures of terms of the type $\left(\gamma_{V_{1}}\right)\left(\gamma_{P_{1}}\right)$, and $\left(\gamma_{V_{2}}\right)\left(\gamma_{P_{2}}\right)$, besides two additional "interference" terms like $\left(\gamma_{V_{1}}\right)\left(\gamma_{P_{2}}\right)$ and $\left(\gamma_{V_{2}}\right)\left(\gamma_{P_{1}}\right)$, where brackets (...) represent the fact that factors like $\frac{\not P}{M} \Lambda^{ \pm \pm}$ and $\Lambda^{ \pm \pm} \frac{\not p}{M}$ sandwich the hadronic Dirac structure $\gamma_{P_{1,2}}$ or $\gamma_{V_{1,2}}$ from left and right, besides the appearance of $P$ and $\ell^{\prime}$ as in Eqs. (28) and (30).

With the use of two Dirac structures, the total amplitude $M_{\mathrm{fi}}$ for the process $P \rightarrow V \gamma$ can be expressed in terms of the transition form factor $F_{P V}$ through Eqs. (39), with detailed expressions given in Eqs. (A6) and (A7) in the Appendix. We can then express the form factor $F_{P V}$ as a superposition of the four amplitudes as $F_{P V}=F_{1}+F_{2}+F_{3}+F_{4}$, where $F_{1}=F_{\gamma_{V_{1}} \gamma_{P_{1}}}, \quad F_{2}=F_{\gamma_{V_{2}} \gamma_{P_{2}}}, \quad F_{3}=F_{\gamma_{V_{1}} \gamma_{P_{2}}}$, and $F_{4}=F_{\gamma_{V_{2}} \gamma_{P_{1}}}$, where the notation $F_{\gamma_{V_{1}} \gamma_{P_{1}}}$ implies the partial amplitude calculated using the mixture of Dirac structures $\left(\gamma_{V_{1}}\right)\left(\gamma_{P_{1}}\right)$ of vector and pseudoscalar mesons. Thus we can at best find the partial amplitudes $F_{i}$, where $i=\gamma_{V_{1}} \gamma_{P_{1}}, \gamma_{V_{2}} \gamma_{P_{2}}, \gamma_{V_{1}} \gamma_{P_{2}}$, and $\gamma_{V_{2}} \gamma_{P_{1}}$ that involve mixtures of these Dirac structures of two different hadrons, and some of the partial amplitudes appear with opposite signs, and their partial contribution to $\Gamma$ is of little relevance. These transition form factors $F_{i}^{\prime} s$ are calculated only at the photon point $\left(k^{2}=0\right)$ where they are real.

Further, the partial contribution of any of these four amplitudes to decay width $\Gamma$ cannot be found, since $\Gamma$ calculated with the use of two Dirac structures will be expressed as $\Gamma \sim\left|F_{P V}\right|^{2}=\left|F_{\gamma_{V_{1}} \gamma_{P_{1}}}\right|^{2}+\left|F_{\gamma_{V_{2}} \gamma_{P_{2}}}\right|^{2}+\left|F_{\gamma_{V_{1}} \gamma_{P_{2}}}\right|^{2}+$ $\left|F_{\gamma_{V_{2}} \gamma_{P_{1}}}\right|^{2}+\sum_{i \neq j} F_{i} F_{j}$, where $i, j=\gamma_{V_{1}} \gamma_{P_{1}}, \gamma_{V_{2}} \gamma_{P_{2}}, \gamma_{V_{1}} \gamma_{P_{2}}$, and $\gamma_{V_{2}} \gamma_{P_{1}}$ and will receive contributions not only from the main diagonal terms but also from the cross terms as well such as $\sum_{i \neq j} F_{i} F_{j}$, each representing mixtures of four different Dirac structures of the two hadrons such as $F_{\gamma_{V_{1}} \gamma_{P_{1}}} F_{\gamma_{V_{2}} \gamma_{P_{2}}}, F_{\gamma_{V_{1}} \gamma_{P_{1}}} F_{\gamma_{V_{1}} \gamma_{P_{2}}}$, etc. Thus, what is of relevance is the sum of these four partial amplitudes to obtain $F_{P V}$, which is directly related to the total width $\Gamma$ as mentioned above.

We list numerical values of the partial amplitude $F_{1}$ along with various subsets of partial amplitudes $F_{1}+F_{2}$, $F_{1}+F_{2}+F_{3}$ and total transition form factor $F_{P V}=F_{1}+$ $F_{2}+F_{3}+F_{4}$ in Table IV when we include two Dirac structures in BS wave functions of the two hadrons. It is seen that amplitudes $F_{1}$ and $F_{2}$ appear with opposite sign in $\eta_{c}(2 S) \rightarrow J / \Psi(1 S) \gamma, D_{S}(2 S) \rightarrow D_{S}^{*}(1 S) \gamma$, and $B_{c}(2 S) \rightarrow$ $B_{c}^{*}(1 S)$, and their magnitudes are of little relevance to their partial contribution to total decay width as explained above. Also the interfering amplitudes that involve mixtures of most leading and lesser leading Dirac structures such as $\gamma_{V_{1}} \gamma_{P_{2}}$ and $\gamma_{V_{2}} \gamma_{P_{1}}$ play an important role when two Dirac structures are taken in both hadronic wave functions.

TABLE IV. Contribution (in $\mathrm{GeV}^{-1}$ ) of partial amplitude $F_{1}$ along with various subsets of partial amplitudes $F_{1}+F_{2}, F_{1}+F_{2}+F_{3}$, and total transition form factor $F_{P V}=F_{1}+F_{2}+F_{3}+F_{4}$ for the processes $P \rightarrow V \gamma$. The total decay width $\Gamma$ for an individual process is in $\mathrm{keV}$.

\begin{tabular}{lcccc}
\hline \hline & $\eta_{c}(2 S) \rightarrow J / \Psi(1 S) \gamma$ & $D(2 S) \rightarrow D^{*}(1 S) \gamma$ & $D_{S}(2 S) \rightarrow D_{S}^{*}(1 S) \gamma$ & $B_{c}(2 S) \rightarrow B_{c}^{*}(1 S) \gamma$ \\
\hline$\Gamma$ & $0.476 \mathrm{keV}$. & $12.157 \mathrm{keV}$ & $7.602 \mathrm{keV}$. & $0.1028 \mathrm{keV}$. \\
$F_{P V}\left({\left.\mathrm{in} \mathrm{GeV}^{-1}\right)}^{-0.006911}\right.$ & -0.0389 & -0.03018 & 0.00521 \\
$F_{1}$ & 0.005227 & -0.0264 & -0.02135 & -0.00421 \\
$F_{1}+F_{2}$ & 0.00191 & -0.0268 & -0.01977 & 0.0154 \\
$F_{1}+F_{2}+F_{3}$ & -0.00411 & -0.03316 & -0.025012 & 0.00520 \\
\hline \hline
\end{tabular}


A similar behavior of partial amplitudes is anticipated for E1 decays.

However, if we make use of the most leading Dirac structures in 4D BS wave functions, as well as the BS normalizers of $V$ and $P$ mesons on the basis of their contributions to leptonic decays [33,37-39,41], we obtain the total transition form factor $F_{P V}=F_{\gamma_{V_{1}} \gamma_{P_{1}}}$, whose magnitude is found to be much higher than $\left|F_{1}\right|$ in Table IV (where all $F_{i}$ 's are evaluated with the use of all Dirac structures in $N_{P}$ and $N_{V}$ ) for all transitions listed, implying thereby that the Dirac structures in BS normalizers also play a crucial role in the calculation of $F_{i}$. The various contributions of partial amplitudes $F_{i}$ to $\Gamma$ seem to be like more of an interference between Dirac structures of two different hadrons. But more detailed investigations on partial contributions of $F_{i}$ for a number of processes involving more than one hadron needs to be done, which to our knowledge has not been studied so far.

In regard to the $E 1$ transitions, we have studied the processes ${ }^{1} P_{1} \rightarrow{ }^{1} S_{0}+\gamma$ that involve the decays $h_{c}(1 P) \rightarrow$ $\eta_{c}(1 S) \gamma, h_{c}(2 P) \rightarrow \eta_{c}(2 S) \gamma$, and $h_{c}(2 P) \rightarrow \eta_{c}(1 S) \gamma$, and the processes ${ }^{1} S_{0} \rightarrow{ }^{1} P_{1}+\gamma$ that involve the decays $\eta_{c}(2 S) \rightarrow h_{c}(1 P) \gamma$. We used algebraic forms of $3 \mathrm{D}$ Salpeter wave functions obtained through analytic solutions of mass spectral equations in an approximate harmonic oscillator basis for ground and excited states of $0^{-+}, 1^{--}$, and $1^{+-}$heavy-light quarkonia for calculation of their decay widths. We have compared our results with experimental data, wherever available, and other models, and found reasonable agreements.

Similarly we again see a wide range of variations in different models for both $M 1$, and $E 1$ transitions, particularly for decays of $\eta_{c}$ and $h_{c}$ mesons. Further, our decay widths for $n S \rightarrow n^{\prime} S$ transitions in $M 1$ decays and $n P \rightarrow n^{\prime} S$ transitions in $E 1$ decays show a marked decrease as we go from ground to higher excited states, which is in conformity with data and other models. We have also given our predictions for radiative decays $h_{c}(2 P) \rightarrow \eta_{c}(2 S) \gamma, h_{c}(2 P) \rightarrow \eta_{c}(1 S) \gamma$, and $\eta_{c}(2 S) \rightarrow h_{c}(1 P)$ for which data are not yet available. In regards to $M 1$ transitions, we have given our prediction for the decay width of $\eta_{c}(2 S) \rightarrow J / \Psi(1 S) \gamma$ for which the PDG tables [12] give only the upper limit on the decay width. Also we calculated the decay width $B_{c}(2 S) \rightarrow B_{c}^{*}(1 S) \gamma$ for which data are not available.

The aim of doing this work was not only to study the processes $P \rightarrow V \gamma, A^{-} \rightarrow P \gamma$, and $P \rightarrow A^{-} \gamma$ for which very little data are available but also to test the algebraic forms of wave functions of $P, V, S, A^{-}$mesons[28,30-32] that we have recently derived from the mass spectral equations of these mesons by following an analytic approach using $4 \times 4$ BSE, whose main features can be found in Refs. [30-32].

A more detailed investigation involving various transitions of $1^{++}$and $1^{+-}$quarkonia, and their transition form factors will be relegated to a separate paper.

\section{ACKNOWLEDGMENTS}

We acknowledge both the Institutions, Chandigarh University and Woldia University for the facilities provided for this work. V. G. would like to acknowledge Chandigarh University for support for her PhD program. We thank the unknown referee for valuable comments that led to the improvements in the manuscript. S. B. would like to thank Shi-Yuan Li (Shandong University, China), and Jorge Mahecha (University of Antioquia, Colombia) for collaborations on earlier works, besides appreciating his continuing association with Addis Ababa University. 


\section{APPENDIX: RADIATIVE TRANSITIONS}

\section{Radiative decays, $P \rightarrow V \gamma$}

Substituting the 3D BS wave function of a pseudoscalar meson in Eq. (35), we obtain the ++ and -- components as

$$
\begin{aligned}
\psi_{P}^{++}(\hat{q})= & \frac{N_{P} \phi_{P}(\hat{q})}{4 \omega_{1} \omega_{2}}\left[M\left(\left(\omega_{1} \omega_{2}+m_{1} m_{2}+\hat{q}^{2}\right)+\left(m_{1} \omega_{2}+\omega_{1} m_{2}\right)\right)-i\left(\left(m_{1} \omega_{2}+\omega_{1} m_{2}\right)+\left(\omega_{1} \omega_{2}+m_{1} m_{2}-\hat{q}^{2}\right)\right) P\right. \\
& \left.+i M\left(\left(\omega_{1}-\omega_{2}\right)+\left(m_{1}-m_{2}\right)\right) \hat{q}-\left(\left(\omega_{1}+\omega_{2}\right)+\left(m_{1}+m_{2}\right)\right) \hat{q} P\right] \gamma_{5}, \\
\psi_{P}^{--}(\hat{q})= & \frac{N_{P} \phi_{P}(\hat{q})}{4 \omega_{1} \omega_{2}}\left[M\left(\left(\omega_{1} \omega_{2}+m_{1} m_{2}+\hat{q}^{2}\right)-\left(m_{1} \omega_{2}+\omega_{1} m_{2}\right)\right)+i\left(\left(m_{1} \omega_{2}+\omega_{1} m_{2}\right)-\left(\omega_{1} \omega_{2}+m_{1} m_{2}-\hat{q}^{2}\right)\right) P\right. \\
& \left.+i M\left(\left(m_{1}-m_{2}\right)-\left(\omega_{1}-\omega_{2}\right)\right) \hat{q}+\left(\left(\omega_{1}+\omega_{2}\right)-\left(m_{1}+m_{2}\right)\right) \hat{\phi} P\right] \gamma_{5} .
\end{aligned}
$$

The adjoint Bethe-Salpeter wave function of a pseudoscalar meson can be obtained by evaluating $\bar{\psi}_{P}^{ \pm \pm}\left(\hat{q}^{\prime}\right)=$ $\gamma_{4}\left(\psi_{P}^{ \pm \pm}\left(\hat{q}^{\prime}\right)\right)^{+} \gamma_{4}$ as

$$
\begin{aligned}
\bar{\psi}_{P}^{++}(\hat{q})= & \frac{N_{P} \phi_{P}(\hat{q})}{4 \omega_{1} \omega_{2}}\left[M\left(\left(\omega_{1} \omega_{2}+m_{1} m_{2}+\hat{q}^{2}\right)+\left(m_{1} \omega_{2}+\omega_{1} m_{2}\right)\right)-i\left(\left(m_{1} \omega_{2}+\omega_{1} m_{2}\right)+\left(\omega_{1} \omega_{2}+m_{1} m_{2}-\hat{q}^{2}\right)\right) P\right. \\
& \left.+i M\left(\left(\omega_{1}-\omega_{2}\right)+\left(m_{1}-m_{2}\right)\right) \hat{\boldsymbol{q}}-\left(\left(\omega_{1}+\omega_{2}\right)+\left(m_{1}+m_{2}\right)\right) P \hat{q}\right] \gamma_{5}, \\
\bar{\psi}_{P}^{--}(\hat{q})= & \frac{N_{P} \phi_{P}(\hat{q})}{4 \omega_{1} \omega_{2}}\left[M\left(\left(\omega_{1} \omega_{2}+m_{1} m_{2}+\hat{q}^{2}\right)-\left(m_{1} \omega_{2}+\omega_{1} m_{2}\right)\right)+i\left(\left(m_{1} \omega_{2}+\omega_{1} m_{2}\right)-\left(\omega_{1} \omega_{2}+m_{1} m_{2}-\hat{q}^{2}\right)\right) P\right. \\
& \left.+i M\left(\left(m_{1}-m_{2}\right)-\left(\omega_{1}-\omega_{2}\right)\right) \hat{\boldsymbol{q}}+\left(\left(\omega_{1}+\omega_{2}\right)-\left(m_{1}+m_{2}\right)\right) P \hat{\boldsymbol{q}}\right] \gamma_{5} .
\end{aligned}
$$

Following the same steps as in the case of pseudoscalar mesons, we obtain the ++ and -- components of a vector meson wave function in Eq. (35) as

$$
\begin{aligned}
& \psi_{V}^{++}\left(\hat{q}^{\prime}\right)=\frac{N_{V} \phi_{V}(\hat{q})}{4 \omega_{1}^{\prime} \omega_{2}^{\prime}}\left[i M^{\prime} \omega_{1}^{\prime} \omega_{2}^{\prime} \notin-\frac{M^{\prime}}{M} \omega_{1}^{\prime} m_{2} \notin P+\frac{i M^{\prime}}{M} \omega_{1}^{\prime} \notin P \hat{q}^{\prime}+\omega_{1}^{\prime} \omega_{2}^{\prime} \notin P^{\prime}+\frac{i m_{2} \omega_{1}^{\prime}}{M} \not P^{\prime} P+\frac{\omega_{1}^{\prime}}{M} \notin P^{\prime} P \hat{q}^{\prime}+\frac{M^{\prime}}{M} \omega_{2}^{\prime} m_{1} P \hat{\ell}\right. \\
& +\frac{i M^{\prime}}{M^{2}} m_{1} m_{2} P \mathscr{\ell} P+\frac{M^{\prime}}{M^{2}} m_{1} P \mathscr{C} P \hat{q}^{\prime}-\frac{i m_{1} \omega_{2}^{\prime}}{M} P \mathscr{E} P^{\prime}+\frac{m_{1} m_{2}}{M^{2}} P \mathscr{\ell} P^{\prime} P-\frac{i m_{1}}{M^{2}} P \mathscr{C} P^{\prime} P \hat{q}^{\prime}-\frac{i M^{\prime}}{M} \omega_{2}^{\prime} \hat{q}^{\prime} P \mathscr{E} \\
& +\frac{M^{\prime}}{M^{2}} m_{2} \hat{q}^{\prime} P \notin P-\frac{i M^{\prime}}{M^{2}} \hat{q}^{\prime} P \notin P \hat{q}^{\prime}-\frac{\omega_{2}^{\prime}}{M} \hat{q}^{\prime} P \notin P^{\prime}-\frac{i m_{2}}{M^{2}} \hat{q}^{\prime} P \notin P^{\prime} P-\frac{1}{M^{2}} \hat{q}^{\prime} P \notin P^{\prime} P \hat{q}^{\prime},
\end{aligned}
$$

$$
\begin{aligned}
& \psi_{V}^{--}\left(\hat{q}^{\prime}\right)=\frac{N_{V} \phi_{V}(\hat{q})}{4 \omega_{1}^{\prime} \omega_{2}^{\prime}}\left[i M^{\prime} \omega_{1}^{\prime} \omega_{2}^{\prime} \notin+\frac{M^{\prime}}{M} \omega_{1}^{\prime} m_{2} \notin P-\frac{i M^{\prime}}{M} \omega_{1}^{\prime} \notin P \hat{q}^{\prime}+\omega_{1}^{\prime} \omega_{2}^{\prime} \notin P^{\prime}-\frac{i m_{2} \omega_{1}^{\prime}}{M} \notin P^{\prime} P-\frac{\omega_{1}^{\prime}}{M} \notin P^{\prime} P \hat{q}^{\prime}-\frac{M^{\prime}}{M} \omega_{2}^{\prime} m_{1} P \ell\right. \\
& +\frac{i M^{\prime}}{M^{2}} m_{1} m_{2} P \mathscr{C} P+\frac{M^{\prime}}{M^{2}} m_{1} P \mathscr{C} P \hat{q}^{\prime}+\frac{i m_{1} \omega_{2}^{\prime}}{M} P \mathscr{\ell} P^{\prime}+\frac{m_{1} m_{2}}{M^{2}} P \mathscr{C} P^{\prime} P-\frac{i m_{1}}{M^{2}} P \mathscr{C} P^{\prime} P \hat{q}^{\prime}+\frac{i M^{\prime}}{M} \omega_{2}^{\prime} \hat{q}^{\prime} P \mathscr{E} \\
& +\frac{M^{\prime}}{M^{2}} m_{2} \hat{q}^{\prime} P \notin P-\frac{i M^{\prime}}{M^{2}} \hat{q}^{\prime} P \notin P \hat{q}^{\prime}+\frac{\omega_{2}^{\prime}}{M} \hat{q}^{\prime} P \notin P^{\prime}-\frac{i m_{2}}{M^{2}} \hat{q}^{\prime} P \notin P^{\prime} P-\frac{1}{M^{2}} \hat{q}^{\prime} P \notin P^{\prime} P \hat{q}^{\prime},
\end{aligned}
$$

where as the adjoint wave functions are 


$$
\begin{aligned}
& \bar{\psi}_{V}^{++}\left(\hat{q}^{\prime}\right)=\frac{N_{V} \phi_{V}(\hat{q})}{4 \omega_{1}^{\prime} \omega_{2}^{\prime}}\left[i M^{\prime} \omega_{1}^{\prime} \omega_{2}^{\prime} \not{\epsilon}-\frac{M^{\prime}}{M} \omega_{1}^{\prime} m_{2} P \mathscr{E}+\frac{i M^{\prime}}{M} \omega_{1}^{\prime} \hat{q}^{\prime} P \mathscr{E}+\omega_{1}^{\prime} \omega_{2}^{\prime} P^{\prime} \not{\epsilon}+\frac{i m_{2} \omega_{1}^{\prime}}{M} P P^{\prime} \not{E}+\frac{\omega_{1}^{\prime}}{M} \hat{q}^{\prime} P P^{\prime} \not{E}+\frac{M^{\prime}}{M} \omega_{2}^{\prime} m_{1} \notin P\right. \\
& +\frac{i M^{\prime}}{M^{2}} m_{1} m_{2} P \notin P+\frac{M^{\prime}}{M^{2}} m_{1} \hat{q}^{\prime} P \notin P-\frac{i m_{1} \omega_{2}^{\prime}}{M} P^{\prime} \in P+\frac{m_{1} m_{2}}{M^{2}} P P^{\prime} \notin P-\frac{i m_{1}}{M^{2}} \hat{q}^{\prime} P P^{\prime} \notin P-\frac{i M^{\prime}}{M} \omega_{2}^{\prime} \notin P \hat{q}^{\prime} \\
& +\frac{M^{\prime}}{M^{2}} m_{2} P \mathscr{C} P \hat{q}^{\prime}-\frac{i M^{\prime}}{M^{2}} \hat{q}^{\prime} P \mathscr{C} P \hat{q}^{\prime}-\frac{\omega_{2}^{\prime}}{M} P^{\prime} \dot{E} P \hat{q}^{\prime}-\frac{i m_{2}}{M^{2}} P P^{\prime} \mathscr{\ell} P \hat{q}^{\prime}-\frac{1}{M^{2}} \hat{q}^{\prime} P P^{\prime} \dot{E} P \hat{q}^{\prime},
\end{aligned}
$$

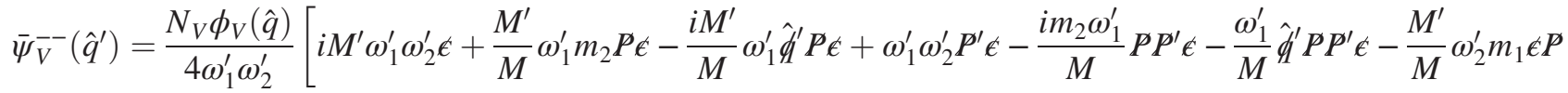

$$
\begin{aligned}
& +\frac{i M^{\prime}}{M^{2}} m_{1} m_{2} P \ell P+\frac{M^{\prime}}{M^{2}} m_{1} \hat{q}^{\prime} P \ell P+\frac{i m_{1} \omega_{2}^{\prime}}{M} P^{\prime} \notin P+\frac{m_{1} m_{2}}{M^{2}} P P^{\prime} \notin P-\frac{i m_{1}}{M^{2}} \hat{q}^{\prime} P P^{\prime} \notin P+\frac{i M^{\prime}}{M} \omega_{2}^{\prime} \notin P \hat{q}^{\prime} \\
& +\frac{M^{\prime}}{M^{2}} m_{2} P \mathscr{\ell} P \hat{q}^{\prime}-\frac{i M^{\prime}}{M^{2}} \hat{q}^{\prime} P \ell P \hat{q}^{\prime}+\frac{\omega_{2}^{\prime}}{M} P^{\prime} \notin P \hat{q}^{\prime}-\frac{i m_{2}}{M^{2}} P P^{\prime} \in P \hat{q}^{\prime}-\frac{1}{M^{2}} \hat{q}^{\prime} P P^{\prime} \in P \hat{q}^{\prime} \text {. }
\end{aligned}
$$

Using the above expressions, we calculate $P \bar{\psi}_{P}^{++}\left(\hat{q}^{\prime}\right) \boldsymbol{\epsilon}^{\prime} \Psi_{V}^{++}(\hat{q}), P \bar{\psi}_{P}^{++}\left(\hat{q}^{\prime}\right) \boldsymbol{\epsilon}^{\prime} \Psi_{V}^{--}(\hat{q}), P \bar{\psi}_{P}^{--}\left(\hat{q}^{\prime}\right) \boldsymbol{\epsilon}^{\prime} \Psi_{V}^{++}(\hat{q})$, and $P \bar{\psi}_{P}^{--}\left(\hat{q}^{\prime}\right) \epsilon^{\prime} \Psi_{V}^{--}(\hat{q})$, which are employed in the calculation of transition form factor $F_{P V}$ for $P \rightarrow V \gamma$. The contribution of $F_{P V}$ from diagram 1 is given by

$$
\begin{aligned}
& F_{P V}^{1}=e_{q} N_{V} N_{P} \frac{1}{M^{2}} \int \frac{d^{3} \hat{q}}{(2 \pi)^{3}} \frac{\phi_{V}\left(\hat{q}^{\prime}\right) \phi_{P}(\hat{q})}{16 \omega_{1} \omega_{2} \omega_{1}^{\prime} \omega_{2}^{\prime}}[T R 1] \\
& {\left[T R_{1}\right]=\left(\alpha_{1} a_{1}+\alpha_{2} b_{1}+\alpha_{3} a_{1}+\alpha_{4} b_{1}\right)\left(\omega_{1}^{\prime} \omega_{2}^{\prime}+M^{\prime} m_{1} \hat{m}_{2}-m_{1} m_{2}-M^{\prime} m_{2} \hat{m}_{2}-\left[-\left(\hat{m}_{1}-\hat{m}_{2} \frac{\left(M^{2}+M^{\prime 2}\right)}{2 M^{2}}\right)^{2} M^{2}\right.\right.} \\
& \left.\left.-\left(\hat{m}_{1}-\hat{m}_{2} \frac{\left(M^{2}+M^{\prime 2}\right)}{2 M^{2}}\right) \hat{m}_{2}\left(M^{2}+M^{\prime 2}\right)-\hat{m}_{2}^{2} M^{\prime 2}\right]\right) \\
& +\left(\alpha_{1} a_{2}+\alpha_{2} b_{2}+\alpha_{3} a_{2}+\alpha_{4} b_{2}\right)\left(-m_{2}\left[\left(\hat{m}_{1}-\hat{m}_{2} \frac{\left(M^{2}+M^{\prime 2}\right)}{2 M^{2}}\right) M^{2}+\hat{m}_{2}\left(M^{2}+M^{\prime 2}\right)\right]\right. \\
& -M^{\prime}\left[\left(\hat{m}_{1}-\hat{m}_{2} \frac{\left(M^{2}+M^{\prime 2}\right)}{2 M^{2}}\right) \hat{m}_{2} M^{2}\right. \\
& \left.\left.+\hat{m}_{2}^{2}\left(M^{2}+M^{\prime 2}\right)\right]-m_{1}\left(\hat{m}_{1}-\hat{m}_{2} \frac{\left(M^{2}+M^{\prime 2}\right)}{2 M^{2}}\right)\left(-M^{2}\right)\right) \\
& +\left(\alpha_{1} a_{3}+\alpha_{2} b_{3}+\alpha_{3} a_{3}+\alpha_{4} b_{3}\right)\left(\omega_{1}^{\prime} \omega_{2}^{\prime} \hat{m}_{2} M^{2}+M^{\prime} m_{1} \hat{m}_{2}^{2}\left(-M^{2}\right)-m_{1} m_{2}\left(-\hat{m}_{2} M^{2}\right)-M^{\prime} M^{2} m_{2} \hat{m}_{2}\right. \\
& \left.-\left[-\left(\hat{m}_{1}-\hat{m}_{2} \frac{\left(M^{2}+M^{\prime 2}\right)}{2 M^{2}}\right)^{2} \hat{m}_{2} M^{4}-\left(\hat{m}_{1}-\hat{m}_{2} \frac{\left(M^{2}+M^{\prime 2}\right)}{2 M^{2}}\right) \hat{m}_{2}^{2}\left(M^{2}+M^{\prime 2}\right) M^{2}+\hat{m}_{2}^{3} M^{2} M^{\prime 2}\right]\right) \\
& +\left(\alpha_{1} a_{4}+\alpha_{2} b_{4}+\alpha_{3} a_{4}+\alpha_{4} b_{4}\right)\left(m_{1}\left(\hat{m}_{1}-\hat{m}_{2} \frac{\left(M^{2}+M^{\prime 2}\right)}{2 M^{2}}\right) \hat{m}_{2} M^{2}+M^{\prime}\left[2\left(\hat{m}_{1}-\hat{m}_{2} \frac{\left(M^{2}+M^{\prime 2}\right)}{2 M^{2}}\right) \hat{m}_{2}^{2} M^{2}\right.\right. \\
& \left.\left.+\hat{m}_{2}^{3}\left(M^{2}+M^{\prime 2}\right)\right]-m_{2}\left[\left(\hat{m}_{1}-\hat{m}_{2} \frac{\left(M^{2}+M^{\prime 2}\right)}{2 M^{2}}\right) M^{2}+\hat{m}_{2}\left(M^{2}+M^{\prime 2}\right)\right]\right) \\
& +\left(-\alpha_{1} a_{1}-\alpha_{2} b_{1}+\alpha_{3} a_{1}+\alpha_{4} b_{1}\right)\left(-M \omega_{1}^{\prime}\left(\hat{m}_{1}-\hat{m}_{2} \frac{\left(M^{2}+M^{\prime 2}\right)}{2 M^{2}}\right)\right. \\
& \left.+\frac{\omega_{2}^{\prime}}{M}\left[\left(\hat{m}_{1}-\hat{m}_{2} \frac{\left(M^{2}+M^{\prime 2}\right)}{2 M^{2}}\right) M^{2}+2 \hat{m}_{2}\left(M^{2}+M^{\prime 2}\right)\right]\right) \\
& +\left(-\alpha_{1} a_{2}-\alpha_{2} b_{2}+\alpha_{3} a_{2}+\alpha_{4} b_{2}\right)\left(-M M^{\prime} \omega_{1}^{\prime} \hat{m}_{2}+M \omega_{1}^{\prime} m_{2}-M m_{1} \omega_{2}^{\prime}-M M^{\prime} \omega_{2}^{\prime} \hat{m}_{2}\right) \\
& +\left(-\alpha_{1} a_{3}-\alpha_{2} b_{3}+\alpha_{3} a_{3}+\alpha_{4} b_{3}\right)\left(\frac{\omega_{2}^{\prime}}{M}\left[\left(\hat{m}_{1}-\hat{m}_{2} \frac{\left(M^{2}+M^{\prime 2}\right)}{2 M^{2}}\right) \hat{m}_{2} M^{4}+\hat{m}_{2}^{2} M^{2}\left(M^{2}+M^{\prime 2}\right)\right]\right. \\
& \left.-M \omega_{1}^{\prime}\left(\hat{m}_{1}-\hat{m}_{2} \frac{\left(M^{2}+M^{\prime 2}\right)}{2 M^{2}}\right) \hat{m}_{2}\left(-M^{2}\right)\right) \\
& +\left(-\alpha_{1} a_{4}-\alpha_{2} b_{4}+\alpha_{3} a_{4}+\alpha_{4} b_{4}\right)\left(M M^{\prime} \omega_{1}^{\prime}\left(-\hat{m}_{2}^{2}\right)-M M^{\prime} \omega_{2}^{\prime} \hat{m}_{2}^{2}-m_{1} \omega_{2}^{\prime} M \hat{m}_{2}-M \omega_{1}^{\prime} m_{2}\left(-\hat{m}_{2}\right)\right) \text {. }
\end{aligned}
$$


Similarly, the contribution of $F_{P V}$ from diagram 2 is given by

$$
\begin{aligned}
& F_{P V}^{2}=e_{Q} N_{V} N_{P} \frac{1}{M^{2}} \int \frac{d^{3} \hat{q}}{(2 \pi)^{3}} \frac{\phi_{V}\left(\hat{q}^{\prime}\right) \phi_{P}(\hat{q})}{16 \omega_{1} \omega_{2} \omega_{1}^{\prime} \omega_{2}^{\prime}}[T R 2] \\
& {\left[T R_{2}\right]=\left(\alpha_{1}^{\prime} a_{1}+\alpha_{2}^{\prime} b_{1}+\alpha_{3}^{\prime} a_{1}+\alpha_{4}^{\prime} b_{1}\right)\left(M^{\prime} m_{1}\left(-\hat{m}_{1}\right)+M^{\prime} m_{2} \hat{m}_{1}+\omega_{1}^{\prime} \omega_{2}^{\prime}-m_{1} m_{2}\right.} \\
& \left.-\frac{1}{M^{2}}\left[\left(-\hat{m}_{2}+\hat{m}_{1} \frac{\left(M^{2}+M^{\prime 2}\right)}{2 M^{2}}\right)^{2} M^{4}+\hat{m}_{1}^{2}\left(M^{2}+M^{\prime 2}\right)^{2}+\hat{m}_{1}^{2} M^{\prime 2} M^{2}\right]\right) \\
& +\left(\alpha_{1}^{\prime} a_{2}+\alpha_{2}^{\prime} b_{2}+\alpha_{3}^{\prime} a_{2}+\alpha_{4}^{\prime} b_{2}\right)\left(-m_{1}\left[-\left(-\hat{m}_{2}+\hat{m}_{1} \frac{\left(M^{2}+M^{\prime 2}\right)}{2 M^{2}}\right) M^{2}+\hat{m}_{1}\left(M^{2}+M^{\prime 2}\right)\right]\right. \\
& \left.-m_{2}\left[-\left(-\hat{m}_{2}+\hat{m}_{1} \frac{\left(M^{2}+M^{\prime 2}\right)}{2 M^{2}}\right) M^{2}+\hat{m}_{1}\left(M^{2}+M^{\prime 2}\right)\right]\right) \\
& +\left(\alpha_{1}^{\prime} a_{3}+\alpha_{2}^{\prime} b_{3}+\alpha_{3}^{\prime} a_{3}+\alpha_{4}^{\prime} b_{3}\right)\left(M^{\prime} m_{1} M^{2} \hat{m}_{1}^{2}-M^{\prime} m_{2} M^{2} \hat{m}_{1}^{2}+\omega_{1}^{\prime} \omega_{2}^{\prime} M^{2}\left(-\hat{m}_{1}\right)+m_{1} m_{2} \hat{m}_{1} M^{2}\right. \\
& -\left[\left(-\hat{m}_{2}+\hat{m}_{1} \frac{\left(M^{2}+M^{\prime 2}\right)}{2 M^{2}}\right)^{2} M^{4}+2\left(-\hat{m}_{2}+\hat{m}_{1} \frac{\left(M^{2}+M^{\prime 2}\right)}{2 M^{2}}\right) \hat{m}_{1}^{2} M^{2}\left(M^{2}+M^{\prime 2}\right)\right. \\
& \left.\left.+\hat{m}_{1}^{3}\left(M^{2}+M^{\prime 2}\right)^{2}+\hat{m}_{1}^{3} M^{\prime 2} M^{2}\right]\right) \\
& +\left(\alpha_{1}^{\prime} a_{4}+\alpha_{2}^{\prime} b_{4}+\alpha_{3}^{\prime} a_{4}+\alpha_{4}^{\prime} b_{4}\right)\left(M^{\prime}\left[\left(-\hat{m}_{2}+\hat{m}_{1} \frac{\left(M^{2}+M^{\prime 2}\right)}{2 M^{2}}\right)\left(-\hat{m}_{1}+\hat{m}_{1}^{2}\right) M^{2}\right]\right. \\
& \left.+m_{2}\left[-\left(-\hat{m}_{2}+\hat{m}_{1} \frac{\left(M^{2}+M^{\prime 2}\right)}{2 M^{2}}\right) M^{2}+\hat{m}_{1}^{2}\left(M^{2}+M^{\prime 2}\right)\right]\right) \\
& +\left(-\alpha_{1}^{\prime} a_{1}-\alpha_{2}^{\prime} b_{1}+\alpha_{3}^{\prime} a_{1}+\alpha_{4}^{\prime} b_{1}\right)\left(-\frac{\omega_{1}^{\prime}}{M}\left[-\left(-\hat{m}_{2}+\hat{m}_{1} \frac{\left(M^{2}+M^{\prime 2}\right)}{2 M^{2}}\right) M^{2}+\hat{m}_{1}\left(M^{2}+M^{\prime 2}\right)\right]\right. \\
& \left.+\frac{\omega_{2}^{\prime}}{M}\left[-\left(-\hat{m}_{2}+\hat{m}_{1} \frac{\left(M^{2}+M^{\prime 2}\right)}{2 M^{2}}\right) M^{2}+\hat{m}_{1}\left(M^{2}+M^{\prime 2}\right)\right]\right) \\
& +\left(-\alpha_{1}^{\prime} a_{2}-\alpha_{2}^{\prime} b_{2}+\alpha_{3}^{\prime} a_{2}+\alpha_{4}^{\prime} b_{2}\right)\left(M M^{\prime} \omega_{2}^{\prime}\left(-\hat{m}_{1}\right)-M m_{2} \omega_{1}^{\prime}+M m_{1} \omega_{2}^{\prime}-M M^{\prime} \omega_{1}^{\prime} \hat{m}_{1}\right) \\
& +\left(-\alpha_{1}^{\prime} a_{3}-\alpha_{2}^{\prime} b_{3}+\alpha_{3}^{\prime} a_{3}+\alpha_{4}^{\prime} b_{3}\right)\left(-M \omega_{1}^{\prime}\left[\left(-\hat{m}_{2}+\hat{m}_{1} \frac{\left(M^{2}+M^{\prime 2}\right)}{2 M^{2}}\right) \hat{m}_{1} M^{2}-\hat{m}_{1}^{2}\left(M^{2}+M^{\prime 2}\right)\right]\right. \\
& \left.+M \omega_{2}^{\prime}\left[\left(-\hat{m}_{2}+\hat{m}_{1} \frac{\left(M^{2}+M^{\prime 2}\right)}{2 M^{2}}\right) \hat{m}_{1} M^{2}-\hat{m}_{1}^{2}\left(M^{2}+M^{\prime 2}\right)\right]\right) \\
& +\left(-\alpha_{1}^{\prime} a_{4}-\alpha_{2}^{\prime} b_{4}+\alpha_{3}^{\prime} a_{4}+\alpha_{4}^{\prime} b_{4}\right)\left(M M^{\prime} \omega_{1}^{\prime} \hat{m}_{1}^{2}-M M^{\prime} \omega_{2}^{\prime}\left(-\hat{m}_{1}^{2}\right)+M m_{2} \omega_{1}^{\prime} \hat{m}_{1}\right. \\
& \left.-m_{1}\left[-\left(-\hat{m}_{2}+\hat{m}_{1} \frac{\left(M^{2}+M^{\prime 2}\right)}{2 M^{2}}\right) \hat{m}_{1} M^{2}+\hat{m}_{1}^{2}\left(M^{2}+M^{\prime 2}\right)\right]-M m_{1} \omega_{2}^{\prime} \hat{m}_{1}\right)
\end{aligned}
$$

where 


$$
\begin{aligned}
& a_{1}=M\left(\left(\omega_{1} \omega_{2}+m_{1} m_{2}+\hat{q}^{2}\right)+\left(m_{1} \omega_{2}+m_{2} \omega_{1}\right)\right), \\
& a_{2}=-\left(m_{1} \omega_{2}+m_{2} \omega_{1}\right)-\left(\omega_{1} \omega_{2}+m_{1} m_{2}-\hat{q}^{2}\right), \\
& a_{3}=-\left(\omega_{1}+\omega_{2}+m_{1}+m_{2}\right), \\
& a_{4}=\left(\omega_{1}-\omega_{2}\right)+\left(m_{1}-m_{2}\right), \\
& b_{1}=M\left(\omega_{1} \omega_{2}+m_{1} m_{2}+\hat{q}^{2}-m_{1} \omega_{2}-m_{2} \omega_{1}\right), \\
& b_{2}=-\left(m_{1} \omega_{2}+\omega_{1} m_{2}\right)+\left(\omega_{1} \omega_{2}+m_{1} m_{2}-\hat{q}^{2}\right), \\
& b_{3}=\left(\omega_{1}+\omega_{2}\right)-\left(m_{1}+m_{2}\right), \\
& b_{4}=-\left(\omega_{1}-\omega_{2}\right)+\left(m_{1}-m_{2}\right) .
\end{aligned}
$$

\section{Radiative decays $\boldsymbol{A}^{-} \rightarrow \boldsymbol{P \gamma}$}

The expressions for the coefficients $a_{i}, b_{i}, d_{i}$, and $e_{i}$ entering into $\Theta_{1}$ and $\Theta_{1}^{\prime}$ in Eqs. (60) and (61) are given as

$$
\begin{aligned}
& a_{1}=-\frac{M^{\prime}}{4}-\frac{m_{1} m_{2} M^{\prime}}{4 \omega_{1}^{\prime} \omega_{2}^{\prime}}-\frac{\hat{q}^{2} M^{\prime}}{4 \omega_{1}^{\prime} \omega_{2}^{\prime}} ; a_{2}=\frac{m_{2} M^{\prime}}{4 \omega_{2}^{\prime} M}+\frac{m_{1} M^{\prime}}{4 \omega_{1}^{\prime} M} ; a_{3}=\frac{1}{4}+\frac{m_{1} m_{2}}{4 \omega_{1}^{\prime} \omega_{2}^{\prime}}+\frac{\hat{q}^{\prime 2}}{4 \omega_{1}^{\prime} \omega_{2}^{\prime}}, \\
& a_{4}=\frac{m_{2}}{4 \omega_{2}^{\prime} ; M}+\frac{m_{1}}{4 \omega_{1}^{\prime} M} ; a_{5}=\frac{M^{\prime}}{4 \omega_{2}^{\prime} M}+\frac{M^{\prime}}{4 \omega_{1}^{\prime} M} ; a_{6}=\frac{-1}{4 \omega_{2}^{\prime} M} ; a_{7}=\frac{M^{\prime} m_{1}}{4 \omega_{1}^{\prime} \omega_{2}^{\prime}}+\frac{M^{\prime} m_{2}}{4 \omega_{1}^{\prime} \omega_{2}^{\prime}}, \\
& a_{8}=\frac{m_{1}}{4 \omega_{1}^{\prime} \omega_{2}^{\prime}}+\frac{m_{2}}{4 \omega_{1}^{\prime} \omega_{2}^{\prime}} ; a_{9}=\frac{-1}{4 \omega_{1}^{\prime} \omega_{2}^{\prime}} ; b_{1}=\frac{M}{4}+\frac{m_{1} m_{2} M}{4 \omega_{1} \omega_{2}}+\frac{m_{1} M}{4 \omega_{1}}+\frac{m_{2} M}{4 \omega_{2}}-\frac{\hat{q}^{2} M}{4 \omega_{1} \omega_{2}}, \\
& b_{2}=\frac{m_{1}}{4 \omega_{1}}+\frac{m_{2}}{4 \omega_{2}}+\frac{1}{4}+\frac{m_{1} m_{2}}{4 \omega_{1} \omega_{2}}-\frac{\hat{q}^{2}}{4 \omega_{1} \omega_{2}} ; b_{3}=-\frac{1}{4 \omega_{1}}+\frac{1}{4 \omega_{2}}-\frac{m_{1}}{4 \omega_{1} \omega_{2}}+\frac{m_{2}}{4 \omega_{1} \omega_{2}}, \\
& b_{4}=-\frac{m_{1} M}{4 \omega_{1} \omega_{2}}-\frac{M}{4 \omega_{2}}+\frac{M}{4 \omega_{1}}-\frac{m_{2} M}{4 \omega_{1} \omega_{2}} ; e_{1}=-\frac{M^{\prime}}{4}-\frac{m_{1} m_{2} M^{\prime}}{4 \omega_{1}^{\prime} \omega_{2}^{\prime}}+\frac{\hat{q}^{\prime 2} M^{\prime}}{4 \omega_{1}^{\prime} \omega_{2}^{\prime}}, \\
& e_{2}=-\frac{m_{2} M^{\prime}}{4 \omega_{2}^{\prime} M}-\frac{m_{1} M^{\prime}}{4 \omega_{1}^{\prime} M} ; e_{3}=\frac{1}{4}-\frac{m_{1} m_{2}}{4 \omega_{1}^{\prime} \omega_{2}^{\prime}}-\frac{\hat{q}^{\prime 2}}{4 \omega_{1}^{\prime} \omega_{2}^{\prime}} ; e_{4}=-\frac{m_{2}}{4 \omega_{2}^{\prime} M}+\frac{m_{1}}{4 \omega_{1}^{\prime} M}, \\
& e_{8}=-\frac{M^{\prime}}{4 \omega_{2}^{\prime} M}-\frac{M^{\prime}}{4 \omega_{1}^{\prime} M} ; e_{6}=-\frac{1}{4 \omega_{2}^{\prime} M} ; e_{7}=\frac{M^{\prime} m_{1}}{4 \omega_{1}^{\prime} \omega_{2}^{\prime}}+\frac{M^{\prime} m_{2}}{4 \omega_{1}^{\prime} \omega_{2}^{\prime}}, \\
& d_{2}=-\frac{m_{1}}{4 \omega_{1}^{\prime}}-\frac{m_{2}}{4 \omega_{2}+\frac{1}{4}}-\frac{m_{1} m_{2}}{4 \omega_{1} \omega_{2}}-\frac{\hat{q}^{2}}{4 \omega_{1} \omega_{2}} ; e_{3}=\frac{1}{4 \omega_{1}^{\prime} M} ; d_{1}=\frac{M}{4}+\frac{m_{1} m_{2} M}{4 \omega_{1} \omega_{2}}-\frac{m_{1} M}{4 \omega_{1}}-\frac{m_{2} M}{4 \omega_{2}}-\frac{\hat{q}^{2} M}{4 \omega_{1} \omega_{2}}, \frac{m_{2}}{4 \omega_{1} \omega_{2}}+\frac{m_{1}}{4 \omega_{1} \omega_{2}}, \\
& d_{4}=\frac{m_{1} M}{4 \omega_{1} \omega_{2}}+\frac{M}{4 \omega_{2}}-\frac{M}{4 \omega_{1}}+\frac{m_{2} M}{4 \omega_{1} \omega_{2}} .
\end{aligned}
$$




\section{Radiative decays $\boldsymbol{P} \rightarrow \boldsymbol{A}^{-} \boldsymbol{\gamma}$}

The coefficients $a_{i}, b_{i}, d_{i}, e_{i}$ entering into $\Delta_{1}$ and $\Delta_{1}^{\prime}$ entering into Eqs. (71) and (72) are given as

$$
\begin{aligned}
& a_{1}^{\prime}=\frac{1}{4 M^{2} \sqrt{\omega_{1} \omega_{2} \omega_{1}^{\prime} \omega_{2}^{\prime}}}\left(M^{3} \omega_{1} \omega_{2}+m_{1} \omega_{2} M^{3}+m_{1} m_{2} M^{3}+\omega_{1} m_{2} M^{3}-M^{3} \hat{q}^{2}\right), \\
& a_{2}^{\prime}=\frac{1}{4 M^{2} \sqrt{\omega_{1} \omega_{2} \omega_{1}^{\prime} \omega_{2}^{\prime}}}\left(M^{2} \omega_{1} \omega_{2}+m_{1} \omega_{2} M^{2}+m_{1} m_{2} M^{2}+\omega_{1} m_{2} M^{2}-M^{2} \hat{q}^{2}\right), \\
& a_{3}^{\prime}=\frac{1}{4 M^{2} \sqrt{\omega_{1} \omega_{2} \omega_{1}^{\prime} \omega_{2}^{\prime}}}\left(-M^{2} \omega_{2}-M^{2} m_{2}+M^{2} \omega_{1}+M^{2} m_{1}\right) ; a_{4}^{\prime}=\frac{1}{4 M^{2} \sqrt{\omega_{1} \omega_{2} \omega_{1}^{\prime} \omega_{2}^{\prime}}}\left(M^{3} \omega_{2}-M^{3} m_{2}-M^{3} m_{1}-M^{3} \omega_{1}\right), \\
& b_{1}^{\prime}=\frac{1}{4 M^{2} \sqrt{\omega_{1} \omega_{2} \omega_{1}^{\prime} \omega_{2}^{\prime}}}\left(-M^{2} M^{\prime} \omega_{1}^{\prime} \omega_{2}^{\prime}-m_{1} m_{2} M^{\prime} M^{2}-M^{2} M^{\prime} \hat{q}^{\prime 2}\right) ; b_{2}^{\prime}=\frac{1}{4 M^{2} \sqrt{\omega_{1} \omega_{2} \omega_{1}^{\prime} \omega_{2}^{\prime}}}\left(m_{1} M^{\prime} M \omega_{2}^{\prime}+m_{2} M^{\prime} M \omega_{1}^{\prime}\right) \text {, } \\
& b_{3}^{\prime}=\frac{1}{4 M^{2} \sqrt{\omega_{1} \omega_{2} \omega_{1}^{\prime} \omega_{2}^{\prime}}}\left(M^{2} \omega_{1}^{\prime} \omega_{2}^{\prime}+M^{2} m_{1} m_{2}+M^{2} \hat{q}^{\prime 2}\right) ; b_{4}^{\prime}=\frac{1}{4 M^{2} \sqrt{\omega_{1} \omega_{2} \omega_{1}^{\prime} \omega_{2}^{\prime}}}\left(M M^{\prime} \omega_{1}^{\prime}+M M^{\prime} \omega_{2}^{\prime}\right), \\
& b_{5}^{\prime}=\frac{1}{4 M^{2} \sqrt{\omega_{1} \omega_{2} \omega_{1}^{\prime} \omega_{2}^{\prime}}}\left(m_{1} M \omega_{2}^{\prime}+m_{2} M \omega_{1}^{\prime}\right) ; b_{6}^{\prime}=\frac{1}{4 M^{2} \sqrt{\omega_{1} \omega_{2} \omega_{1}^{\prime} \omega_{2}^{\prime}}}\left(-M \omega_{1}^{\prime}\right), \\
& b_{7}^{\prime}=\frac{1}{4 M^{2} \sqrt{\omega_{1} \omega_{2} \omega_{1}^{\prime} \omega_{2}^{\prime}}}\left(M^{2} M^{\prime}\left(m_{2}+m_{1}\right) ; \quad b_{8}^{\prime}=\frac{1}{4 M^{2} \sqrt{\omega_{1} \omega_{2} \omega_{1}^{\prime} \omega_{2}^{\prime}}} M^{2}\left(m_{1}+m_{2}\right) ; b_{9}^{\prime}=\frac{1}{4 M^{2} \sqrt{\omega_{1} \omega_{2} \omega_{1}^{\prime} \omega_{2}^{\prime}}}\left(-M \omega_{2}^{\prime}\right)\right. \text {, } \\
& e_{1}^{\prime}=\frac{1}{4 M^{2} \sqrt{\omega_{1} \omega_{2} \omega_{1}^{\prime} \omega_{2}^{\prime}}} M^{2}\left(-M^{\prime} \omega_{1}^{\prime} \omega_{2}^{\prime}-m_{1} m_{2} M^{\prime}+M^{\prime} \hat{q}^{2}\right) ; \quad e_{2}^{\prime}=\frac{1}{4 M^{2} \sqrt{\omega_{1} \omega_{2} \omega_{1}^{\prime} \omega_{2}^{\prime}}} M^{\prime} M\left(-m_{1} \omega_{2}^{\prime}-m_{2} \omega_{1}^{\prime}\right), \\
& e_{3}^{\prime}=\frac{1}{4 M^{2} \sqrt{\omega_{1} \omega_{2} \omega_{1}^{\prime} \omega_{2}^{\prime}}} M^{2}\left(\omega_{1}^{\prime} \omega_{2}^{\prime}-m_{1} m_{2}-\hat{q}^{\prime 2}\right) ; d_{4}^{\prime}=\frac{1}{4 M^{2} \sqrt{\omega_{1} \omega_{2} \omega_{1}^{\prime} \omega_{2}^{\prime}}} M^{3}\left(\omega_{1}-\omega_{2}+m_{2}+m_{1}\right), \\
& e_{4}^{\prime}=\frac{1}{4 M^{2} \sqrt{\omega_{1} \omega_{2} \omega_{1}^{\prime} \omega_{2}^{\prime}}} M M^{\prime}\left(\omega_{1}^{\prime}-\omega_{2}^{\prime}\right) ; \quad e_{5}^{\prime}=\frac{1}{4 M^{2} \sqrt{\omega_{1} \omega_{2} \omega_{1}^{\prime} \omega_{2}^{\prime}}} M\left(m_{1} \omega_{2}^{\prime}-m_{2} \omega_{1}^{\prime}\right) ; \quad e_{6}^{\prime}=\frac{1}{4 M^{2} \sqrt{\omega_{1} \omega_{2} \omega_{1}^{\prime} \omega_{2}^{\prime}}}\left(-M \omega_{1}^{\prime}\right) \text {, } \\
& e_{7}^{\prime}=\frac{1}{4 M^{2} \sqrt{\omega_{1} \omega_{2} \omega_{1}^{\prime} \omega_{2}^{\prime}}} M^{\prime} M^{2}\left(m_{1}+m_{2}\right) ; e_{8}^{\prime}=\frac{1}{4 M^{2} \sqrt{\omega_{1} \omega_{2} \omega_{1}^{\prime} \omega_{2}^{\prime}}} M^{2}\left(-m_{1}-m_{2}\right) ; e_{9}^{\prime}=\frac{1}{4 M^{2} \sqrt{\omega_{1} \omega_{2} \omega_{1}^{\prime} \omega_{2}^{\prime}}}\left(M \omega_{2}^{\prime}\right) \text {, } \\
& d_{1}^{\prime}=\frac{1}{4 M^{2} \sqrt{\omega_{1} \omega_{2} \omega_{1}^{\prime} \omega_{2}^{\prime}}} M^{3}\left(\omega_{1} \omega_{2}-m_{1} \omega_{2}+m_{1} m_{2}-\omega_{1} m_{2}-\hat{q}^{2}\right) ; d_{3}^{\prime}=\frac{1}{4 M^{2} \sqrt{\omega_{1} \omega_{2} \omega_{1}^{\prime} \omega_{2}^{\prime}}} M^{2}\left(\omega_{2}-\omega_{1}+m_{1}-m_{2}\right), \\
& d_{2}^{\prime}=\frac{1}{4 M^{2} \sqrt{\omega_{1} \omega_{2} \omega_{1}^{\prime} \omega_{2}^{\prime}}} M^{2}\left(-m_{1} \omega_{2}+\omega_{1} \omega_{2}-\omega_{1} m_{2}-m_{1} m_{2}-\hat{q}^{2}\right) .
\end{aligned}
$$

[1] J. S. Whitaker et al., Phys. Rev. Lett. 37, 1596 (1976).

[2] C. J. Biddick et al., Phys. Rev. Lett. 38, 1324 (1977).

[3] C. Klopfenstein et al. (CUSB Collaboration), Phys. Rev. Lett. 51, 160 (1983).

[4] F. Pauss et al. (CUSB Collaboration), Phys. Lett. 130B, 439 (1983).

[5] P. Haas et al. (CLEO Collaboration), Phys. Rev. Lett. 52, 799 (1984).

[6] C. Baglin et al., Phys. Lett. B 171, 135 (1986).

[7] T. A. Armstrong et al., Phys. Rev. Lett. 69, 2337 (1992).

[8] M. Andreotti et al., Phys. Rev. D 72, 032001 (2005).

[9] J. L. Rosner et al. (CLEO Collaboration), Phys. Rev. Lett. 95, 102003 (2005).
[10] M. Ablikim et al. (BESIII Collaboration), Phys. Rev. Lett. 118, 092002 (2017).

[11] K. A. Olive et al. (Particle Data Group Collaboration), Chin. Phys. C 38, 090001 (2014).

[12] P. A. Zyla et al. (Particle Data Group Collaboration), Prog. Theor. Exp. Phys. (2020), $083 \mathrm{C} 01$.

[13] N. Brambila et al., arxiv:hep-ph/0412158.

[14] E. Eichten, S. Godfrey, H. Mahlke, and J. L. Rosner, Rev. Mod. Phys. 80, 1161 (2008).

[15] N. Brambila, Y. Jia, and A. Vairo, Phys. Rev. D 73, 054005 (2006).

[16] A. Pineda and J. Segovia, Phys. Rev. D 87, 074024 (2013). 
[17] M. Li, Y. Li, P. Maris, and J. P. Vary, Phys. Rev. D 98, 034024 (2018).

[18] H. M. Choi, Phys. Rev. D 75, 073016 (2007).

[19] Y. L. Shi, Eur. Phys. J. C 77, 253 (2017).

[20] D. Becirevic and F. Sanfillipo, J. High Energy Phys. 01 (2013) 028.

[21] G. C. Donald, C. T. H.Davies, R. J. Dowdall, E. Follana, K. Hornbostel, J. Koponen, G. P. Lepage, and C. Mc Neile, Phys. Rev. D 86, 094501 (2012).

[22] A. N. Mitra and B. M. Sodermark, Nucl. Phys. A695, 328 (2001).

[23] J. Carbonell and V. A. Karmanov, Phys. Rev. D 91, 076010 (2015).

[24] J. K. He and C. J. Fan, Phys. Rev. D 103, 114006 (2021).

[25] T. Wang, Y. Jiang, W. L. Ju, H. Yuan, and G. L.Wang, J. High Energy Phys. 03 (2016) 209.

[26] S. Bhatnagar and E. Gebrehana, Phys. Rev. D 102, 094024 (2020).

[27] W. J. Deng, H. Liu, L. C. Gui, and X. H. Zhong, Phys. Rev. D 95, 034026 (2017).

[28] V. Guleria and S. Bhatnagar, Int. J. Theor. Phys. 60, 3143 (2021).

[29] A. N. Mitra and S. Bhatnagar, Int. J. Mod. Phys. A 07, 121 (1992).

[30] H. Negash and S. Bhatnagar, Int. J. Mod. Phys. E 25, 1650059 (2016).

[31] S. Bhatnagar and L. Alemu, Phys. Rev. D 97, 034021 (2018).

[32] E. Gebrehana, S. Bhatnagar, and H. Negash, Phys. Rev. D 100, 054034 (2019).

[33] S. Bhatnagar, J. Mahecha, and Y. Mengesha, Phys. Rev. D 90, 014034 (2014).

[34] H. Negash and S. Bhatnagar, Adv. High Energy Phys. 2017, 7306825 (2017).

[35] C. H. Chang, J. K. Chen, and G. L. Wang, Commun. Theo. Phys.(Beijing) 46, 467 (2006).

[36] C. H. L. Smith, Ann. Phys. (N.Y.) 53, 521 (1969).
[37] R. Alkofer and L. V. Smekel, Phys. Rep. 353, 281 (2002).

[38] S. Bhatnagar and S-Y. Li, J. Phys. G 32, 949 (2006).

[39] S. Bhatnagar, S-Y. Li, and J. Mahecha, Int. J. Mod. Phys. E 20, 1437 (2011).

[40] H. J. Munczek and P. Jain, Phys. Rev. D 46, 438 (1992); P. Jain and H. J. Munczek, Phys. Rev. D 48, 5403 (1993).

[41] V. Sauli, Phys. Rev. D 86, 096004 (2012).

[42] T. Kawanai and S. Sasaki, AIP Conf. Proc. 1701, 050022 (2016).

[43] T. Burch, C. DeTar, M. Di Pierro, A. X. El-Khadra, E. D. Freeland, S. Gottlieb, A. S. Kronfeld, L. Levkova, P. B. Mackenzie, and J. N. Simone, Phys. Rev. D 81, 034508 (2010).

[44] M. Shah, B. Patel, and P. C. Vinodkumar, Phys. Rev. D 90, 014009 (2014).

[45] C. T. H. Davies, K. Hornbostel, G. P. Lepage, J. Shigemitsu, and J. H. Sloan, Phys. Lett. B 382, 131 (1996).

[46] P. P. Souza, A. P. Momterio, and K. B. Vijaya Kumar, arXiv:1703.10413.

[47] D. Ebert, R. N. Faustov, and V. O. Galkin, Phys. At. Nucl. 76, 1554 (2013).

[48] D. Ebert, R. N. Faustov, and V. O. Galkin, Phys. Rev. D 67, 014027 (2003).

[49] W. J. Deng, H. Liu, L. C. Gui, and X. H. Zhong, Phys. Rev. D 95, 034026 (2017).

[50] T. Barnes, S. Godfrey, and E. S. Swanson, Phys. Rev. D 72, 054026 (2005).

[51] V. H. Kher and A. K. Rai, J. Phys. 934, 012036 (2017).

[52] V. V. Kiselev, A. K. Likhoed, and A. V. Tkabladze, Phys. Rev. D 51, 3613 (1995).

[53] E. J. Eichten and C. Quigg, Phys. Rev. D 49, 5845 (1994).

Correction: Proof alterations to remove paragraphs (a) and (b) and to insert explanatory text at the end of the penultimate paragraph in Sec. VI were overlooked and have now been implemented. 